\title{
Differential operators and the wheels power series
}

\author{
ANDREW KRICKER
}

\begin{abstract}
An earlier work of the author's showed that it was possible to adapt the AlekseevMeinrenken Chern-Weil proof of the Duflo isomorphism to obtain a completely combinatorial proof of the wheeling isomorphism. That work depended on a certain combinatorial identity, which said that a particular composition of elementary combinatorial operations arising from the proof was precisely the wheeling operation. The identity can be summarized as follows: The wheeling operation is just a graded averaging map in a space enlarging the space of Jacobi diagrams. The purpose of this paper is to present a detailed and self-contained proof of this identity. The proof broadly follows similar calculations in the Alekseev-Meinrenken theory, though the details here are somewhat different, as the algebraic manipulations in the original are replaced with arguments concerning the enumerative combinatorics of formal power series of graphs with graded legs.
\end{abstract}

17B99, 57M25; 05E99

The Duflo isomorphism is a Lie theoretic result from the 1970s which says that if $\mathfrak{g}$ is a finite-dimensional Lie algebra then $(\mathcal{S} \mathfrak{g})^{\mathfrak{g}}$, the ring of $\mathfrak{g}$-invariants in the symmetric algebra on $\mathfrak{g}$, and $(\mathcal{U} \mathfrak{g})^{\mathfrak{g}}$, the ring of $\mathfrak{g}$-invariants in the universal enveloping algebra of $\mathfrak{g}$, are isomorphic as rings. Duflo explicitly constructed the isomorphism to be the result of applying a certain infinite order differential operator to $(\mathcal{S} \mathfrak{g})^{\mathfrak{g}}$ followed by an application of the averaging map $(\mathcal{S} \mathfrak{g})^{\mathfrak{g}} \rightarrow(\mathcal{U} \mathfrak{g})^{\mathfrak{g}}$.

The wheeling isomorphism is a combinatorial strengthening of the Duflo isomorphism. The algebras $(\mathcal{U} \mathfrak{g})^{\mathfrak{g}}$ and $(\mathcal{S} \mathfrak{g})^{\mathfrak{g}}$ are replaced by $\mathcal{A}$ and $\mathcal{B}$, combinatorially constructed algebras generated by certain abstract unitrivalent graphs known as Jacobi diagrams. Duflo's infinite differential operator is replaced by the wheeling operation, $\partial_{\Omega}: \mathcal{B} \rightarrow \mathcal{B}$, in which one glues a certain formal power series of "Wheel" graphs into the given element, in "all possible ways".

Wheeling was discovered at the end of the last century in the study of the Kontsevich integral knot invariant; see Bar-Natan, Garoufalidis, Rozansky and Thurston [4] and Bar-Natan, Le and Thurston [6]. It has since found many applications beyond those already covered by the Duflo isomorphism, not only in quantum topology (such as Garoufalidis and Kricker [8]), but, for example, to such surprising topics as the theory of hyper-Kahler manifolds (as in Roberts and Willerton [13] and Nieper-Wißkirchen [12]). 
At first glance, the operation which appears in the statement of the wheeling isomorphism $-\chi_{\mathcal{B}} \circ \partial_{\Omega}: \mathcal{B} \rightarrow \mathcal{A}$ - does not seem to be a particularly natural operation. The purpose of this paper is to provide a detailed proof of an identity which says that $\chi_{\mathcal{B}} \circ \partial_{\Omega}$ can be factored into a sequence of elementary combinatorial operations. In summary: wheeling is just a graded averaging map in a space which enlarges $\mathcal{A}$.

\section{The main identity and the spaces and maps involved in it}

First we'll state the identity in question. After that (beginning in Section 1.1) we'll go through the definitions of the various spaces and maps that are involved in the identity. In Section 2 we'll outline the organization of the proof.

Main Theorem Consider $\chi_{\mathcal{B}} \circ \partial_{\Omega}: \mathcal{B} \rightarrow \mathcal{A}$, the operation which appears in the statement of the wheeling isomorphism. This map can be factored into a sequence of elementary combinatorial operations. To be precise, the composition of maps

$$
\mathcal{B} \stackrel{\partial_{\Omega}}{\longrightarrow} \mathcal{B} \stackrel{\chi_{\mathcal{B}}}{\longrightarrow} \mathcal{A} \stackrel{\phi_{\mathcal{A}}}{\longrightarrow} \widehat{\mathcal{W}}_{\wedge},
$$

is equal to the composition

$$
\mathcal{B} \stackrel{\Upsilon}{\longrightarrow} \mathcal{W} \stackrel{\chi_{\mathcal{W}}}{\longrightarrow} \widetilde{\mathcal{W}} \stackrel{\pi}{\longrightarrow} \widehat{\mathcal{W}} \stackrel{B_{\bullet} \rightarrow \mathrm{F}}{\longrightarrow} \widehat{\mathcal{W}}_{\mathrm{F}} \stackrel{\lambda}{\longrightarrow} \widehat{\mathcal{W}}_{\wedge}
$$

Each of the spaces in the above theorem will be defined as a certain "space of diagrams": a $\mathbb{Q}$-vector space consisting of formal, finite $\mathbb{Q}$-linear combinations of abstract graphs with vertices of degree 1 and degree 3 , modulo certain relations which depend on the specific space. In this precise form such spaces first emerged in the theory of finite-type knot invariants (see Birman and Lin [7], Kontsevich [9] and Bar-Natan [3]), though their roots go much deeper. From different points of view they can been seen as developing from the Feynman diagrams of Quantum Field Theory, from the universal combinatorics of commutator expressions in the theory of metrized Lie algebras, and from the combinatorics of the finite-type filtration of the space of knots.

The specific context of the identity is its application in the paper [11] of the author to a combinatorial proof of wheeling.

More generally, this identity is interesting for the following reason: The wheeling operation (to be recalled shortly) is a bit mysterious as it requires a nontrivial power series (the Duflo series $(1 / 2) \log (\sinh (h / 2) /(h / 2)))$ to be inserted "by hand" into the definition, and because it uses wheels, which are relatively complicated subgraphs to be working with. On the other hand, each of the operations that this identity factors 
it into $-\Upsilon, \chi_{\mathcal{W}}$ and $\lambda$ are the nontrivial steps - has a natural definition in terms of some simple combinatorial operation you can perform on the generating graphs, "in all possible ways".

\subsection{The space $\mathcal{B}$ of symmetric Jacobi diagrams}

Each of the spaces involved in the statement of the main theorem is constructed in a similar way, as a "space of diagrams". We'll take the first space $\mathcal{B}$ as our model, and describe its construction in detail.

A symmetric Jacobi diagram is a finite multigraph with vertices of degree 1 and degree 3. By "multigraph" we are formally allowing parallel edges and "loops" (ie edges whose endpoints coincide). Each trivalent vertex with three distinct incident edges is "oriented", which means that the set of incident edges is given a cyclic ordering. When we draw a symmetric Jacobi diagram in the plane, we give the trivalent vertices the counterclockwise orientation induced by the drawing.

The space $\mathcal{B}$ is defined to be the space of formal finite $\mathbb{Q}$-linear combinations of symmetric Jacobi diagrams, modulo two classes of relations, the "AS relations", and the "IHX relations". The AS relations (for antisymmetry) say that when we change the orientation of a trivalent vertex we pick up a minus sign. For example,

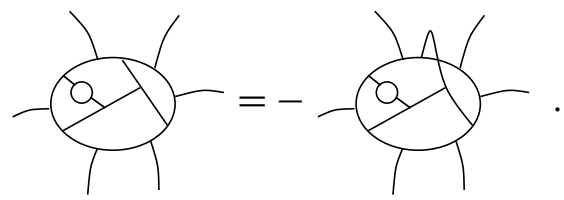

The relation which sets a Jacobi diagram with a "loop" (in the sense just referred to) to be zero will also be regarded as an AS relation.

The IHX relation is as follows:

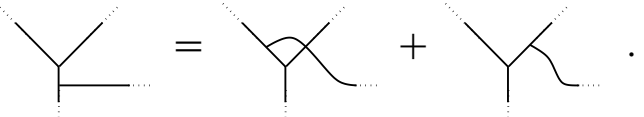

Here we are referring to three diagrams which are exactly the same, except that they differ in the shown subgraph.

The connection with the theory of metrized Lie algebras via "weight systems" is the most direct way to get some intuition for these relations (see eg Bar-Natan [3]). 


\subsection{The space $\mathcal{A}$ of ordered Jacobi diagrams}

In the definition of the space $\mathcal{A}$, symmetric Jacobi diagrams are replaced with ordered Jacobi diagrams. In an ordered Jacobi diagram the degree 1 vertices are given a total ordering. This ordering is recorded in drawings by lining up the degree 1 vertices along an ordering line which is drawn at the bottom of the diagram. In this paper we will frequently refer to the degree 1 vertices as the legs of the diagram.

In the space $\mathcal{A}$, we take $\mathbb{Q}$-linear combinations of ordered Jacobi diagrams modulo AS, IHX and an extra relation which concerns the legs, the "STU" relation, which is as follows:

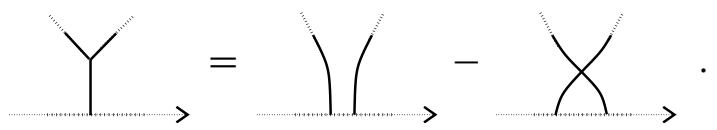

\subsection{The averaging map $\chi_{\mathcal{B}}: \mathcal{B} \rightarrow \mathcal{A}$}

The averaging map $\chi_{\mathcal{B}}$ is the linear extension of the map which maps a symmetric Jacobi diagram in $\mathcal{B}$ to the average of all the possible ordered Jacobi diagrams obtained by ordering the degree 1 vertices. For example,

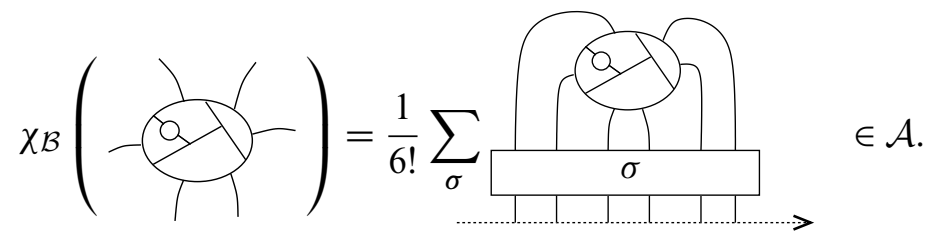

It turns out that while this map is an isomorphism of vector spaces (this is the formal Poincaré-Birkhoff-Witt theorem described in [3]), it is not an isomorphism of algebras. In other words, if $u$ and $v$ are elements of $\mathcal{B}$ then it is not in general true that $\chi_{\mathcal{B}}(u \sqcup$ $v)=\chi_{\mathcal{B}}(u) \# \chi_{\mathcal{B}}(v)$, where $\sqcup$ is the "disjoint-union" product on $\mathcal{B}$, and \# is the "juxtaposition" product on $\mathcal{A} .^{1}$

\subsection{The wheeling map $\partial_{\Omega}: \mathcal{B} \rightarrow \mathcal{B}$}

The wheeling operation $\partial_{\Omega}: \mathcal{B} \rightarrow \mathcal{B}$ is a linear isomorphism of $\mathcal{B}$ which promotes $\chi_{\mathcal{B}}$ to an algebra isomorphism:

$$
\left(\chi_{\mathcal{B}} \circ \partial_{\Omega}\right)(u \sqcup v)=\left(\chi_{\mathcal{B}} \circ \partial_{\Omega}\right)(u) \#\left(\chi_{\mathcal{B}} \circ \partial_{\Omega}\right)(v)
$$

for all $u, v \in \mathcal{B}$.

${ }^{1}$ A comment on notation: In situations like this, where we have a map, like the averaging map, which we use several different versions of, we'll distinguish the different versions by writing the domain of the map as a subscript. Hence: $\chi_{\mathcal{B}}$. 
Bar-Natan, Le and Thurston's [6] proof of wheeling shows that it is deeply bound up with the theory of the Kontsevich integral, and such things as the theory of associators, the monodromy of the Knizhnik-Zamolodchikov equations, and the theory of quantum groups. The aim of this paper, together with its companion [11], is to describe a completely combinatorial proof of the wheeling isomorphism, with the goal of discovering new approaches to these topics. This proof derives from work of Alekseev and Meinrenken [1;2], as is discussed in the author's paper [11].

So, what is this map $\partial_{\Omega}$ ? First we must recall what is $\partial_{X}(Y) \in \mathcal{B}$, the result of operating on a symmetric Jacobi diagram $Y$ with a symmetric Jacobi diagram $X$. The result is the sum of all the possible symmetric Jacobi diagrams that you obtain by gluing all of the legs of $X$ to some (possibly all) of the legs of $Y$. This is extended linearly to define $\partial_{u}(v)$, for arbitrary $u, v \in \mathcal{B}$.

In the case that $X$ has more legs than $Y, \partial_{X}(Y)$ will be zero. This means that it meaningful to consider operations of the form $\partial_{\mathcal{P}}: \mathcal{B} \rightarrow \mathcal{B}$, where $\mathcal{P}$ is an infinite combination of symmetric Jacobi diagrams (a "formal power series of diagrams"), as long as for each $b \in \mathbb{N}$, the piece of $\mathcal{P}$ consisting of the diagrams whose number of degree 1 vertices is less than $b$ is finite. (These issues are carefully discussed, from a more general viewpoint, in Section 3.)

To recall $\Omega$, the power series appearing in wheeling, we'll use the following convenient notation for generating a formal power series of symmetric Jacobi diagrams:

denotes

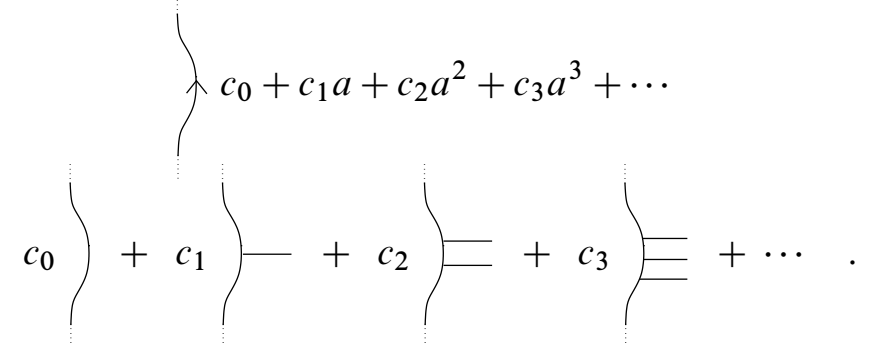

The following precise statement uses $\mathcal{B}^{n}$, which is the subspace of $\mathcal{B}$ generated by diagrams with precisely $n$ degree 1 vertices.

Definition 1.4.1 The wheels element, $\Omega$, is the formal power series of symmetric Jacobi diagrams defined by the expression

$$
\Omega=\exp _{\sqcup}\left(\frac{1}{2} \bigcirc \ln \left(\frac{\sinh a / 2}{a / 2}\right)\right) \in \prod_{n=0}^{\infty} \mathcal{B}^{n} .
$$




\subsection{The space $\mathcal{W}$}

In the earlier work [11], $\mathcal{W}$ was introduced as an " $\iota$-complex", which was a pair of cochain complexes equipped with a degree -1 map between them. In this work we have no need for all this extra structure, and $\mathcal{W}$ just denotes the vector space underlying the structure.

The diagrams which generate $\mathcal{W}$, which will be called symmetric Weil diagrams in this paper, have degree 1 vertices of two different types. There are "leg-grade 1" vertices, which are drawn without any decoration, and "leg-grade 2" vertices, which are drawn with a fat dot. The space $\mathcal{W}$ consists of formal finite $\mathbb{Q}$-linear combinations of Weil diagrams, modulo AS and IHX relations, and also relations which say that when we transpose the position of two adjacent legs in the ordering, we pick up a sign $(-1)^{x y}$, where $x$ and $y$ are the leg-grades of the involved legs.

So, for example, the following equations hold in $\mathcal{W}$ :

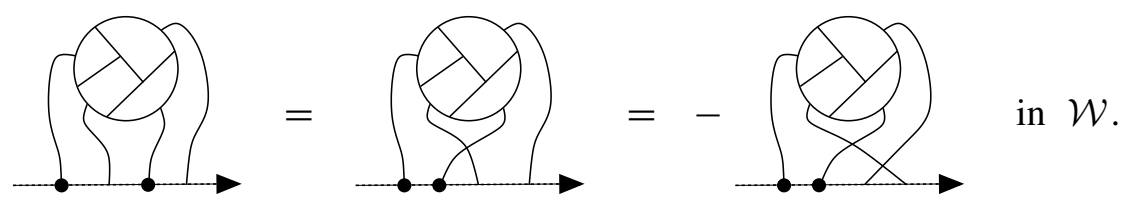

Observe the arrow-head with which symmetric Weil diagrams are drawn.

\subsection{The hair-splitting map $\Upsilon: \mathcal{B} \rightarrow \mathcal{W}$}

Now we'll recall the map $\Upsilon$ which embeds $\mathcal{B}$ into the space $\mathcal{W}$. On some symmetric Jacobi diagram $v$, the map is just to choose an ordering of the degree 1 vertices of $v$ (sometimes called the "hair" of $v$ ), and then to replace each degree 1 vertex according to the rule

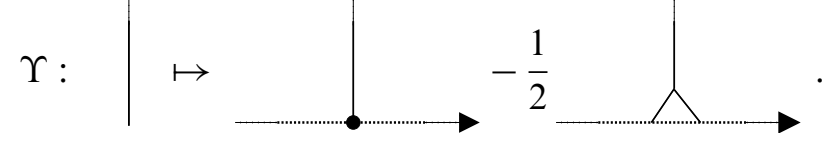

So, for example,

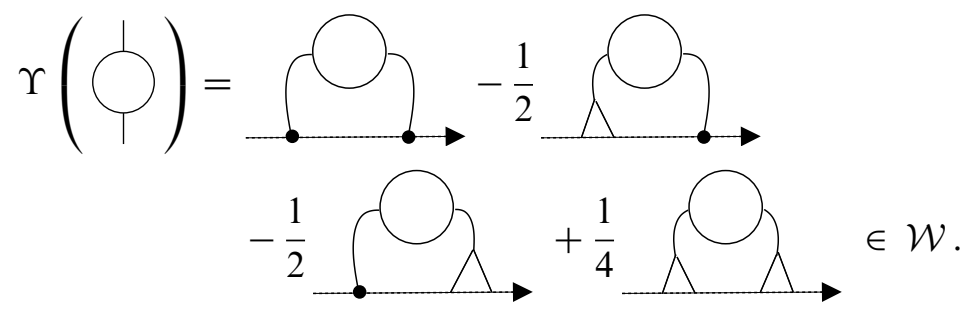




\subsection{The graded averaging map $\chi_{\mathcal{W}}: \mathcal{W} \rightarrow \widetilde{\mathcal{W}}$}

The space $\widetilde{\mathcal{W}}$ is defined in exactly the same way as the space $\mathcal{W}$, but without introducing the leg transposition relations. So the relationship between $\mathcal{W}$ and $\widetilde{\mathcal{W}}$ is analogous to the relationship between the symmetric algebra and the tensor algebra on some vector space. The diagrams which generate this space will be called "noncommutative Weil diagrams" in this paper.

We can embed $\mathcal{W}$ into $\widetilde{\mathcal{W}}$ by means of the graded averaging map $\chi_{\mathcal{W}}: \mathcal{W} \rightarrow \widetilde{\mathcal{W}}$. This is the linear extension of the map which takes a symmetric Weil diagram to the average of all possible rearrangements of the legs of the diagram, accompanied by the sign that arises when that permutation is performed in $\mathcal{W}$. For example,

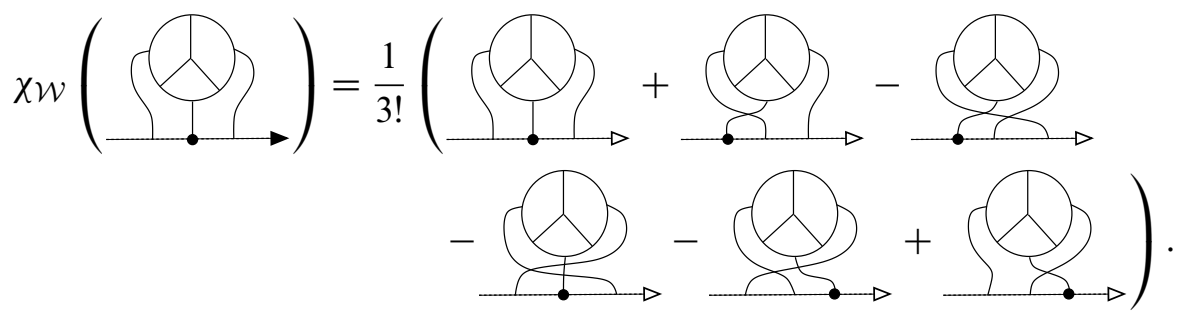

The reader can check that this map respects leg transposition relations.

\subsection{The space $\widehat{\mathcal{W}}$ and the map $\pi: \widetilde{\mathcal{W}} \rightarrow \widehat{\mathcal{W}}$}

Noncommutative Weil diagrams have no relations that relate different orderings of their legs. But the space we are heading towards $-\mathcal{A}-$ has STU relations, so we had better introduce them. As discussed in [11] (deriving from work of Alekseev and Meinrenken), when we introduce STU relations amongst the leg-grade 2 legs, there are some other classes of relations that we must introduce at the same time, so as to retain the algebraic structure of an $\iota$-complex.

The complete set of relations that we introduce when we introduce STU is shown in Figure 1.

Observe that the third class presented above is a formal analogue of the defining relation of a Clifford algebra. The map $\pi: \widetilde{\mathcal{W}} \rightarrow \widehat{\mathcal{W}}$ is just to introduce these relations, with no other effect on a diagram (other than to change the style of the arrowhead).

\subsection{Curvature legs and the map $B_{\bullet \rightarrow F}: \widehat{\mathcal{W}} \rightarrow \widehat{\mathcal{W}}_{\mathrm{F}}$}

Instead of the usual leg-grade 2 legs that have appeared in the discussion up to this point (the legs drawn with a fat dot), it is possible to work with a different choice of 

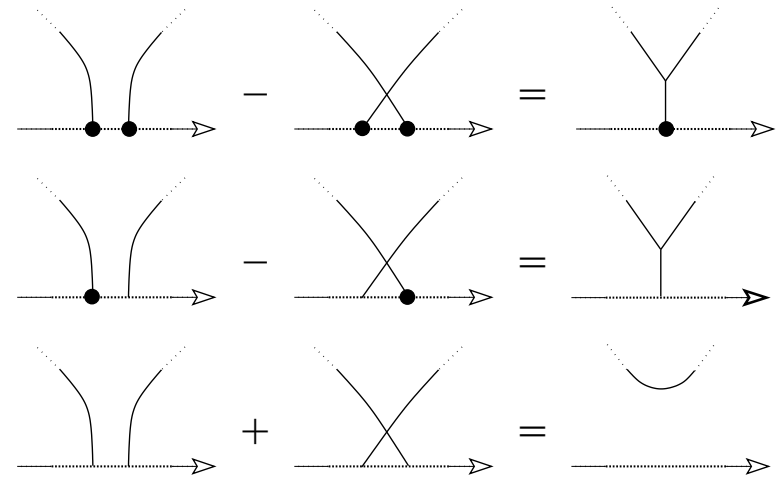

Figure 1: The relations amongst legs in $\widehat{\mathcal{W}}$

leg-grade 2 leg, which we'll call curvature legs in this work. The relationship between the two choices can be expressed by the equation (with arrow-head appropriate to the space):

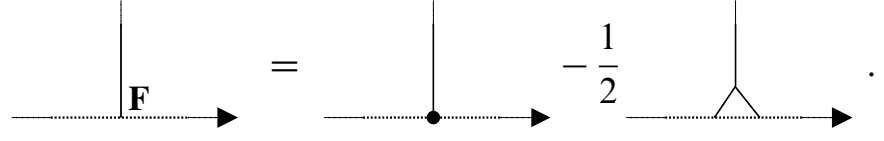

In the algebraic theory, this is just a different choice of generators within a common algebra. In the current work, from the combinatorial point of view, it is clearer to view diagrams that are based on curvature legs as generators of a different, though isomorphic, vector space, and the above equality should only be viewed heuristically. Curvature legs are introduced into the theory to simplify the map $\iota$, though at the expense of a more complicated differential.

If we base the space $\widehat{\mathcal{W}}$ on curvature legs, instead of the usual leg-grade 2 legs, we are led to the space $\widehat{\mathcal{W}}_{\mathrm{F}}$, which has the relations shown in Figure 2.

The "change of basis" map, $\mathrm{B}_{\bullet \rightarrow \mathrm{F}}: \widehat{\mathcal{W}} \rightarrow \widehat{\mathcal{W}}_{\mathrm{F}}$, is just to replace every leg-grade 2 leg with a curvature leg, via the operation

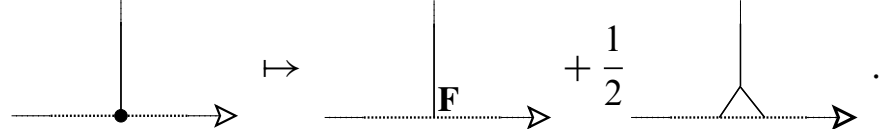

\subsection{The space $\widehat{\mathcal{W}}_{\wedge}$}

The final space to discuss is the space $\widehat{\mathcal{W}}_{\wedge}$. This space consists of formal finite $\mathbb{Q}-$ linear combinations of diagrams with leg-grade 1 legs and curvature legs (ie exactly 


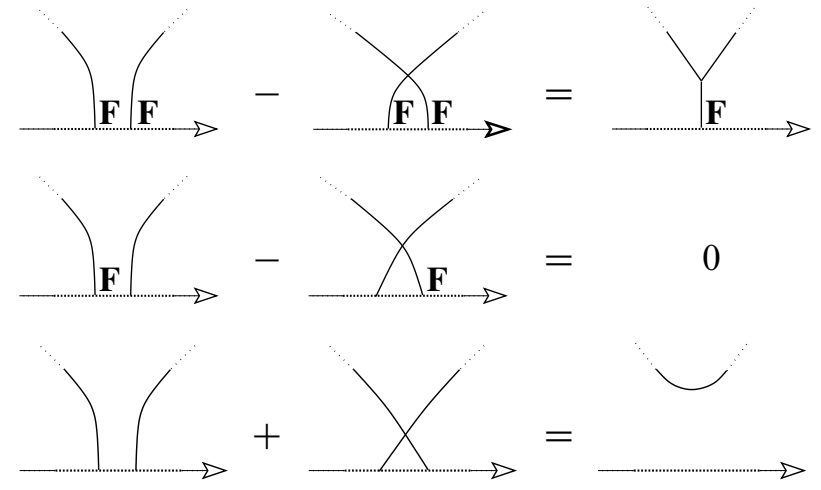

Figure 2: The relations amongst legs in $\widehat{\mathcal{W}}_{\mathrm{F}}$

the same diagrams as is used by $\widehat{\mathcal{W}}_{\mathrm{F}}$ ), taken modulo exactly the same relations as the space $\widehat{\mathcal{W}}_{\mathrm{F}}$ except that the "Clifford" relation (amongst the grade 1 legs) is replaced by the following relation:

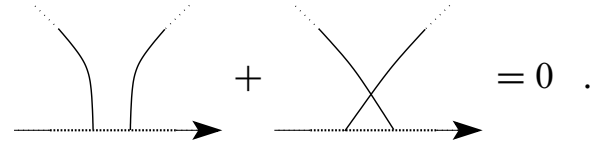

Observe that in this space the leg-grade 1 legs can be moved about freely, up to sign. In particular, this space is graded by the number of leg-grade 1 legs that a diagram has.

This space can be thought of as $\widehat{\mathcal{W}}_{\mathrm{F}}$ viewed with respect to generators in which the leg-grade 1 legs have been symmetrized. To be precise: we have a well-defined map, $\chi_{\wedge}: \widehat{\mathcal{W}}_{\wedge} \rightarrow \widehat{\mathcal{W}}_{\mathrm{F}}$, which graded averages the leg-grade 1 legs. An example is shown in Figure 3 .

Just like the case $\chi_{\mathcal{B}}$, which is the formal PBW isomorphism considered in [3], the map $\chi_{\wedge}$ is a vector space isomorphism. However, something is true in this case which is not true for $\chi_{\mathcal{B}}$ : the inverse map has an elementary construction.

\subsection{The map $\lambda: \widehat{\mathcal{W}}_{\mathrm{F}} \rightarrow \widehat{\mathcal{W}}_{\wedge}$}

Here we'll recall the definition of the map $\lambda$ which inverts $\chi_{\wedge}$. See [11] for proofs that it is well-defined, and actually inverts $\chi_{\wedge}$.

The definition can be summarized in the following way: Glue the grade 1 legs to each other in all possible ways, with appropriate coefficients. To be precise, recall that a pairing of the grade 1 legs of a diagram $w$ is a collection, possibly empty, of disjoint 


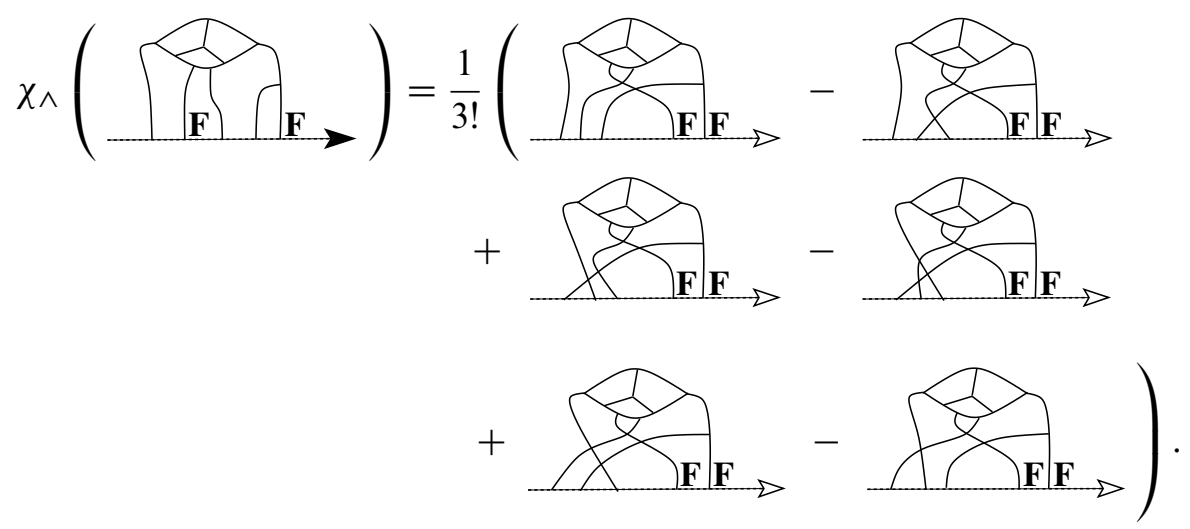

Figure 3: An example of the computation of $\chi_{\wedge}$

2-element subsets of the set of grade 1 legs of $w$. Let $\mathcal{P}(w)$ denote the set of pairings of the diagram $w$. Then $\lambda$ is defined as the linear extension of the map which sends a diagram $w$ to a certain sum

$$
\lambda(w)=\sum_{\wp \in \mathcal{P}(w)} \mathcal{D}_{\wp}(w),
$$

where $\mathcal{D}_{\wp}(w)$ denotes $w$ with its grade 1 legs glued together according to the pairing $\wp$, equipped with an appropriate coefficient. To recall the exact coefficient, we'll follow through the following example:

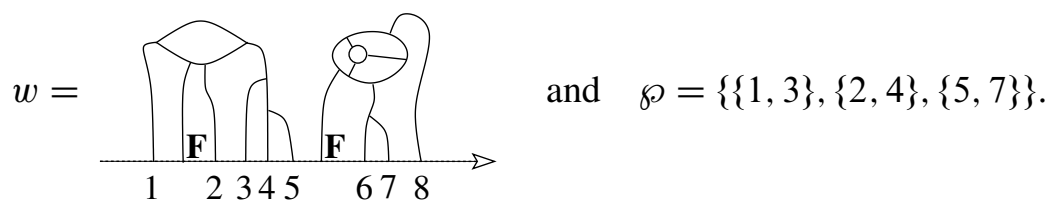

Begin by introducing a second orienting line underneath the diagram, with a gap separating the two orienting lines. Then, for every pair of legs in the pairing $\wp$, add an arc, using a full line, between the corresponding legs of the diagram (such that the introduced arc has no self-intersections and stays within the gap between the two orienting lines). Finally, carry all the remaining legs straight down onto the bottom orienting line, using a full line for the grade 1 legs and a dashed line for the grade 2 legs:

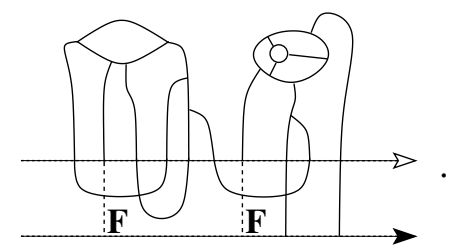


Let $x$ denote the number of intersections between full lines displayed within the gap. The term $\mathcal{D}_{\wp}(w)$ is this diagram (with the original orienting line forgotten and the dashed lines filled in) multiplied by $(-1)^{x}$ and one factor of $\left(\frac{1}{2}\right)$ for every pair of legs glued together.

Thus, in the example at hand,

$$
\mathcal{D}_{\{\{1,3\},\{2,4\},\{5,7\}\}}=(-1)^{2}\left(\frac{1}{2}\right)^{3}
$$

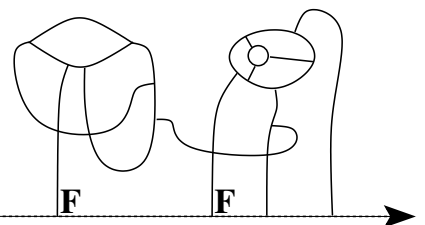

Figure 4 gives an example of the result of a full calculation of $\lambda$.
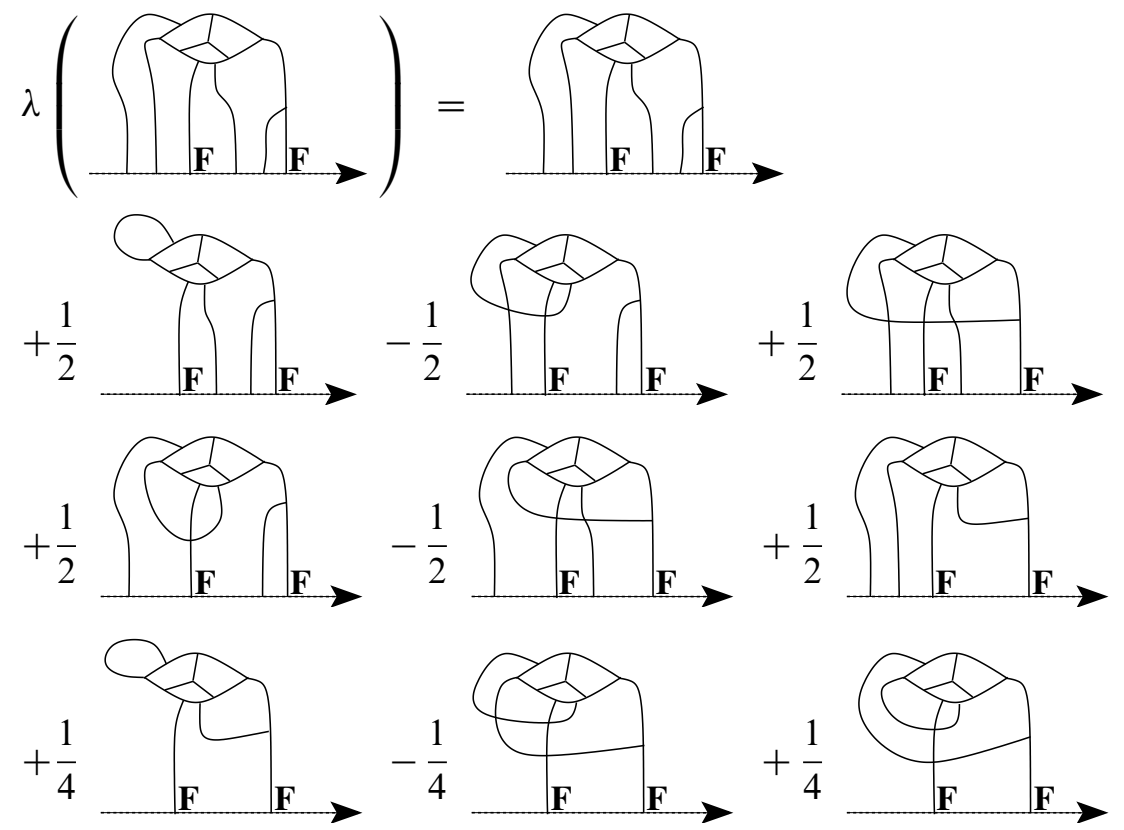

Figure 4: An example of the calculation of $\lambda$

\subsection{The map $\phi_{\mathcal{A}}: \mathcal{A} \rightarrow \widehat{\mathcal{W}}_{\wedge}$}

Observe that the space $\widehat{\mathcal{W}}_{\wedge}$ has no relations which involve the leg-grade 1 legs, except relations which say that when we transpose an adjacent pair of such legs then we pick 
up a minus sign. This means that $\widehat{\mathcal{W}}_{\wedge}$ is graded by the number of leg-grade 1 legs that a diagram has

$$
\widehat{\mathcal{W}}_{\wedge} \cong \bigoplus_{i=0} \widehat{\mathcal{W}}_{\wedge}^{i}
$$

where $\widehat{\mathcal{W}}_{\wedge}^{i}$ denotes the subspace generated by diagrams with exactly $i$ leg-grade 1 legs. The space $\widehat{\mathcal{W}}_{\wedge}^{0}$ is clearly isomorphic to $\mathcal{A}$, and $\phi_{\mathcal{A}}: \mathcal{A} \rightarrow \widehat{\mathcal{W}}_{\wedge}$ is the corresponding embedding, whose action on an ordered Jacobi diagram is simply to make every leg a curvature leg, for example,

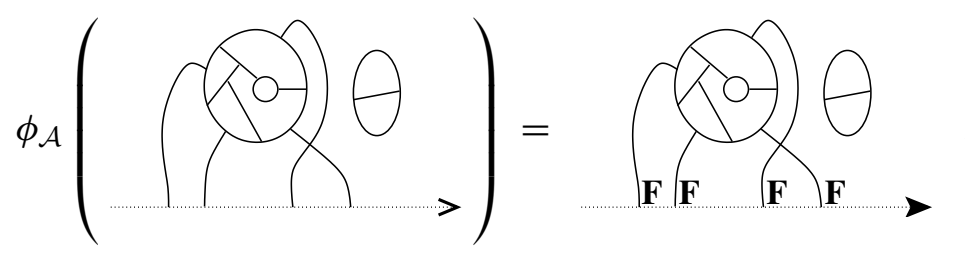

\section{Outline of the contents of this paper}

The fact that this sequence of elementary combinatorial operations is just the wheeling operation $\phi_{\mathcal{A}} \circ \chi_{\mathcal{B}} \circ \partial_{\Omega}$ may be a little surprising. Just how do these maps lead to wheels being glued into legs?

In Figure 5 we illustrate the mechanism which produces wheels by taking a random symmetric Jacobi diagram and following it as it maps through this composition. At each stage in the composition we have drawn a diagram that is typical of the diagrams appearing in the sum at that point. Observe that the last diagram is equal to the image under $\phi_{\mathcal{A}}$ of

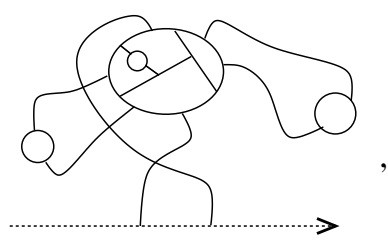

the original diagram with two wheels glued into its legs and its remaining legs given some ordering.

This shows that it is immediate that the composition $\lambda \circ \mathrm{B}_{\bullet \rightarrow \mathrm{F}} \circ \pi \circ \chi_{\mathcal{W}} \circ \Upsilon$ produces the same sort of diagrams as the wheeling operation, $\phi_{\mathcal{A}} \circ \chi_{\mathcal{B}} \circ \partial_{\Omega}$, (as well as some other diagrams). Showing that the diagrams are produced with the correct coefficients, and that all other diagrams produced cancel, is the difficulty of the proof.

The paper is organized in the following way: 


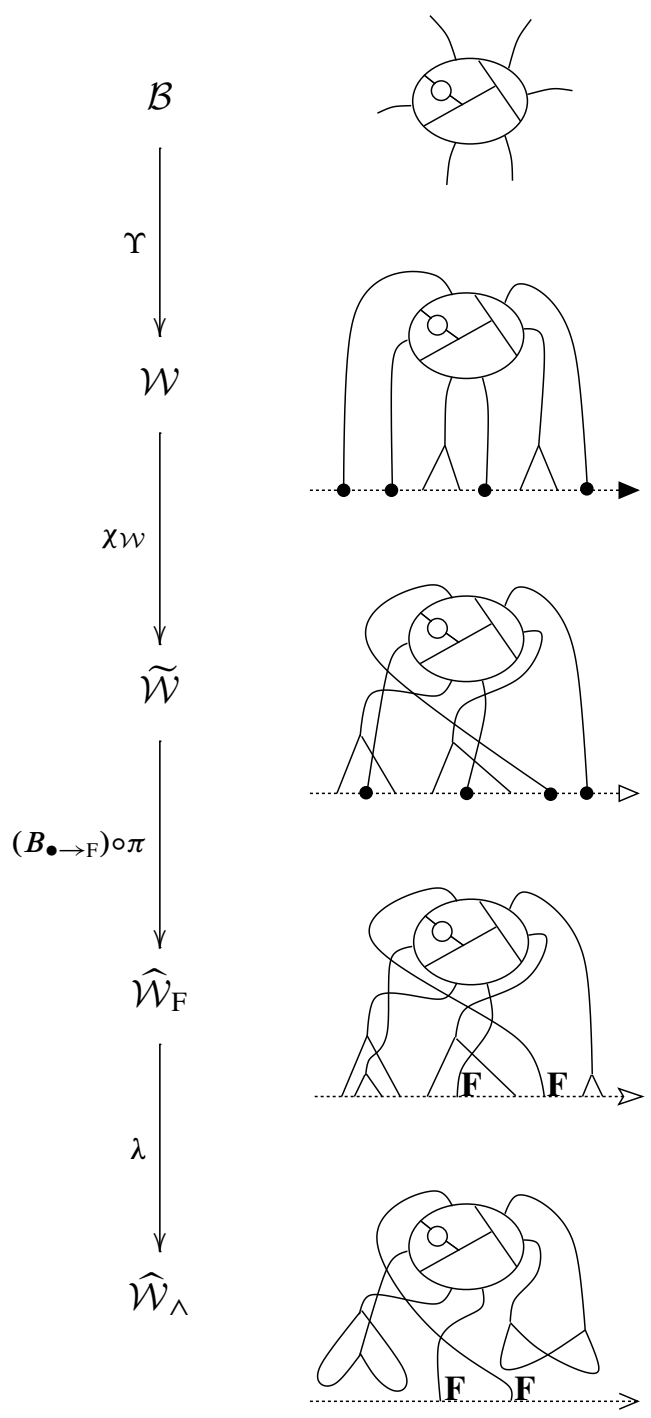

Figure 5: An illustration of how "wheels" appear

In Section 3 we explain how formal power series of diagrams of various sorts operate on each other. In the case that the legs are ungraded this is a familiar operation, being, for example, the calculus in which the Århus 3-manifold invariant is constructed by Bar-Natan et al [5].

In Section 4 we develop a certain expression (Theorem 4.1.1) for the composition $\lambda \circ B_{\bullet} \rightarrow \mathbf{F} \circ \pi \circ \chi_{\mathcal{W}} \circ \Upsilon$ in terms of these diagram operations. The key idea is that we 
can (graded) symmetrize a diagram by turning it into a differential operator and then applying it to a suitable formal exponential (Proposition 4.3.2).

The remaining two sections (Sections 5 and 6) do a direct calculation of the expression developed in Theorem 4.1.1. The key idea in these computations is that the result of an exponential of connected diagrams operating on another exponential of connected diagrams is the exponential of all the connected diagrams you can construct from the logarithms of the pieces. This is a familiar story in the ungraded case - we provide detailed proofs that it holds in certain cases of the graded setting as well. So all we need to do is calculate all the possible connected diagrams that can be constructed. This turns out to be a manageable combinatorial problem in the cases that arise.

Acknowledgements The author thanks Dror Bar-Natan and Eckhard Meinrenken for their support of the author at the University of Toronto during the most important part of this work.

\section{Operating with diagrams on diagrams}

\subsection{Operator Weil diagrams}

We'll now begin to operate on Weil diagrams with other Weil diagrams. To introduce this formalism we'll build a vector space $\widehat{\mathcal{W}}_{\mathbf{F}} \llbracket a, b, \partial_{a}, \partial_{b} \rrbracket$. (The constructions to follow adapt in an unambiguous way to build spaces like $\widehat{\mathcal{W}}_{\wedge} \llbracket a, b, \partial_{a}, \partial_{b} \rrbracket$, etc.) Intuitively: we are taking the vector space $\widehat{\mathcal{W}}_{\mathbf{F}}$, adjoining a formal grade 2 variable $a$ and a formal grade 1 variable $b$ and their corresponding differential operators, and then taking power series with respect to those introduced symbols.

Formally: this space will be built from diagrams which may have the usual legs for $\widehat{\mathcal{W}}_{\mathbf{F}}$, but which may have, in addition, two types of parameter legs and their corresponding operator legs:

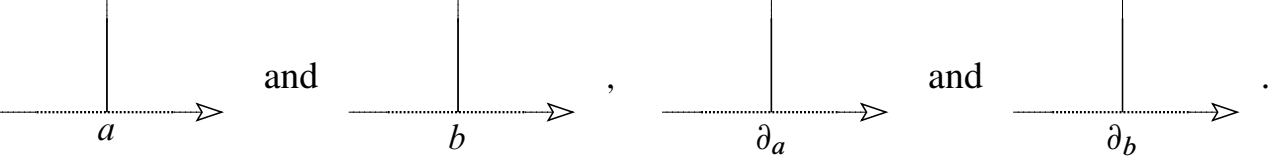

We require that the operator legs appear in a group at the far right-hand end of the diagram. The parameter legs may appear amongst the usual legs in any order.

Let the parameter-grade of a diagram be the total grade of its parameter legs, where $a$-labelled legs count for 2 and $b$-labelled legs count for 1 . The diagram above has parameter-grade 3 . Similarly define the quantity operator-grade; the diagram above 
has operator-grade 4 . If a diagram has parameter-grade $i$ and operator-grade $j$ then we say that it is an $(i, j)$-operator Weil diagram. The pair $(i, j)$ will be referred to as the type of the diagram.

Definition 3.1.1 Define the vector space $\widehat{\mathcal{W}}_{\mathbf{F}}\left[a, b, \partial_{a}, \partial_{b}\right]^{(i, j)}$ to be the space of formal $\mathbb{Q}$-linear combinations of operator Weil diagrams of type $(i, j)$, subject to the same relations that the space $\widehat{\mathcal{W}}_{\mathbf{F}}$ uses, together with relations that say that the parameter and operator legs can be moved about freely (up to the appropriate sign), as long as the operator legs all stay at the far-right hand end of the orienting line.

For example, the following equations hold in $\widehat{\mathcal{W}}_{\mathbf{F}}\left[a, b, \partial_{a}, \partial_{b}\right]^{(3,2)}$ :
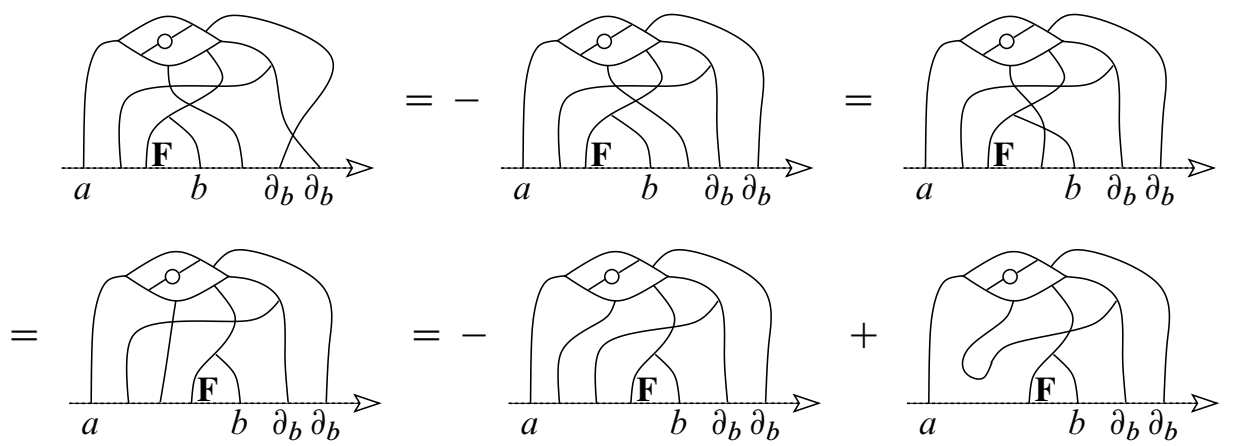

We will work with power series of operator Weil diagrams. Here is what we mean:

Definition 3.1.2 Define the space of formal power series of operator Weil diagrams in the following way:

$$
\widehat{\mathcal{W}}_{\mathbf{F}} \llbracket a, b, \partial_{a}, \partial_{b} \rrbracket=\prod_{(i, j) \in \mathbb{N}_{0} \times \mathbb{N}_{0}} \widehat{\mathcal{W}}_{\mathbf{F}}\left[a, b, \partial_{a}, \partial_{b}\right]^{(i, j)},
$$

where $\mathbb{N}_{0}$ denotes the set of nonnegative integers.

\subsection{The operator pairing}

We will now introduce a bilinear pairing on these power series:

$$
\vdash: \widehat{\mathcal{W}}_{\mathbf{F}} \llbracket a, b, \partial_{a}, \partial_{b} \rrbracket \times^{\prime} \widehat{\mathcal{W}}_{\mathbf{F}} \llbracket a, b, \partial_{a}, \partial_{b} \rrbracket \rightarrow \widehat{\mathcal{W}}_{\mathbf{F}} \llbracket a, b, \partial_{a}, \partial_{b} \rrbracket .
$$

The notation $x^{\prime}$ is to record the fact that the pairing is only defined (only "converges") on certain pairs of power series:

$$
\widehat{\mathcal{W}}_{\mathbf{F}} \llbracket a, b, \partial_{a}, \partial_{b} \rrbracket \times^{\prime} \widehat{\mathcal{W}}_{\mathbf{F}} \llbracket a, b, \partial_{a}, \partial_{b} \rrbracket \subset \widehat{\mathcal{W}}_{\mathbf{F}} \llbracket a, b, \partial_{a}, \partial_{b} \rrbracket \times \widehat{\mathcal{W}}_{\mathbf{F}} \llbracket a, b, \partial_{a}, \partial_{b} \rrbracket .
$$

The discussion below requires the projection map

$$
\operatorname{pr}^{(i, j)}: \widehat{\mathcal{W}}_{\mathbf{F}} \llbracket a, b, \partial_{a}, \partial_{b} \rrbracket \rightarrow \widehat{\mathcal{W}}_{\mathbf{F}}\left[a, b, \partial_{a}, \partial_{b}\right]^{(i, j)} .
$$


3.2.1 How to operate with a diagram The purpose of operator Weil diagrams, of course, is to have them operate on each other. We'll first define how individual diagrams operate on each other, and then extend that action to power series. Consider, then, two operator Weil diagrams:
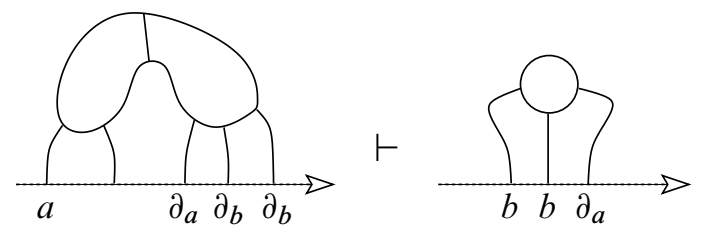

To operate with the first on the second, you begin by placing the two diagrams adjacent to each other on the orienting line. Then you proceed to push the operator legs to the far-right hand side of the resulting diagram by using substitution rules which declare that the operator legs act as graded differential operators. To be precise: if the operator leg encounters a parameter leg corresponding to the same parameter, then it operates on that leg:

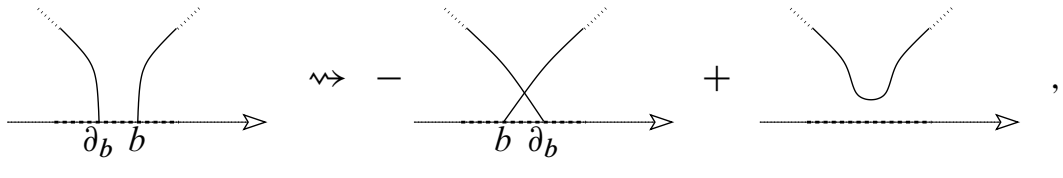

or
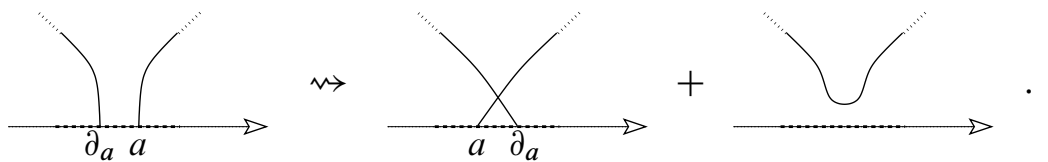

If, on the other hand, the operator leg encounters any leg it is not matched to, then the operator is just pushed past the leg, incurring the appropriate sign $\left((-1)^{g_{1} g_{2}}\right.$, where $g_{1}$ and $g_{2}$ are the leg-grades of the two legs involved). Figure 6 illustrates such a computation. The reader might like to check that they get the final result, which is contained in Figure 7.

3.2.2 Checking relations We have just defined the operation $\vdash$ on the level of diagrams. The following proposition, whose proof is routine and easy-to-check diagrammatics, says that this operation respects the many different relations that exist amongst the legs of diagrams.

Proposition 3.2.3 Let $i, j, k$ and $l$ be elements of $\mathbb{N}_{0}$. The linear extension of the above definition of $\vdash$ gives a well-defined bilinear map

$$
\vdash: \widehat{\mathcal{W}}_{\mathbf{F}}\left[a, b, \partial_{a}, \partial_{b}\right]^{(i, j)} \times \widehat{\mathcal{W}}_{\mathbf{F}}\left[a, b, \partial_{a}, \partial_{b}\right]^{(k, l)} \rightarrow \widehat{\mathcal{W}}_{\mathbf{F}} \llbracket a, b, \partial_{a}, \partial_{b} \rrbracket .
$$

A detailed proof that the relations are respected appeared in the first version of this paper [10]. 

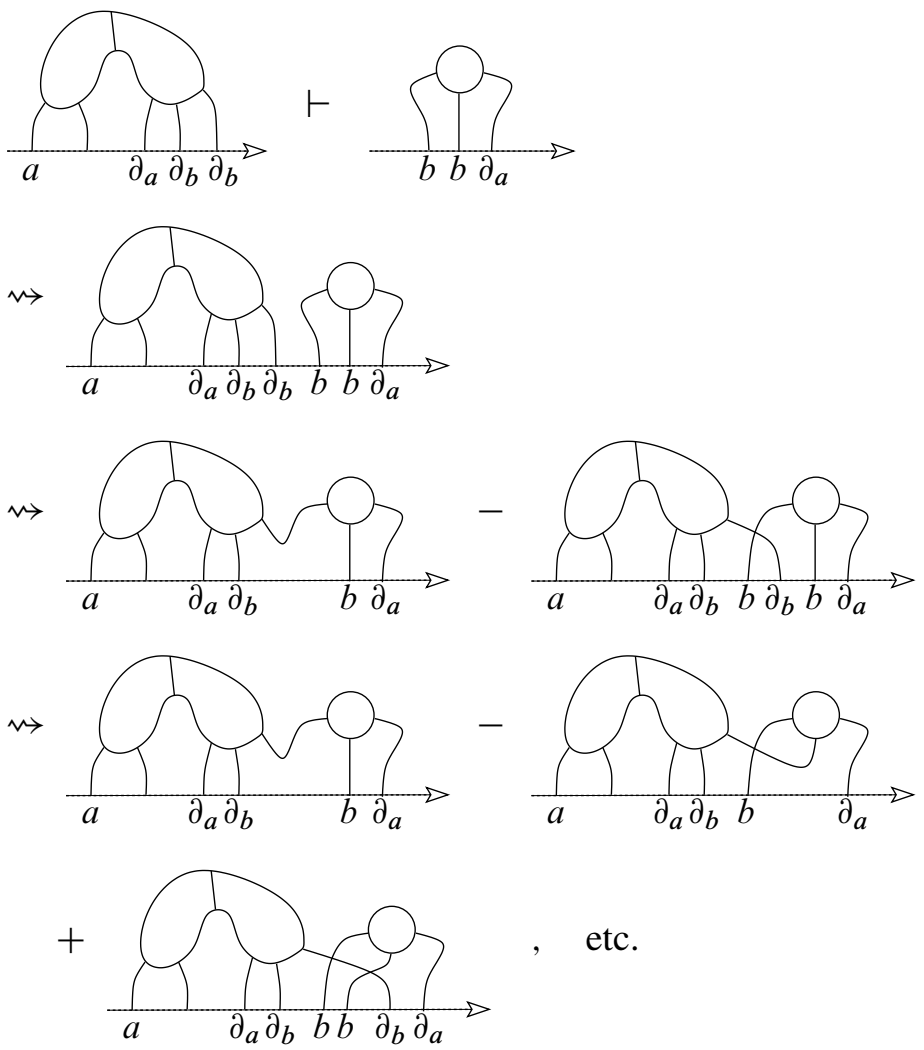

Figure 6: An illustration of one diagram operating on another

3.2.4 The extension to power series Recall that we are working with "formal power series" of Weil operator diagrams, which are a choice, for every pair $(i, j)$ of nonnegative integers, of a vector from $\widehat{\mathcal{W}}_{\mathbf{F}}[a, b]^{(i, j)}$ :

$$
\widehat{\mathcal{W}}_{\mathbf{F}} \llbracket a, b, \partial_{a}, \partial_{b} \rrbracket=\prod_{(i, j) \in \mathbb{N}_{0} \times \mathbb{N}_{0}} \widehat{\mathcal{W}}_{\mathbf{F}}\left[a, b, \partial_{a}, \partial_{b}\right]^{(i, j)} .
$$

In this section we will extend the operation product $\vdash$ to these power series in the obvious way: to multiply two power series, do it term-by-term, then add up the results. Because there is the possibility of infinite sums coming out of this, we must be careful making statements in generality about this product.

For a power series $v$ write

$$
v=\sum_{(i, j) \in \mathbb{N}_{0} \times \mathbb{N}_{0}} v^{(i, j)},
$$

where $v^{(i, j)}=\operatorname{pr}^{(i, j)}(v)$, the type $(i, j)$ piece of $v$. 

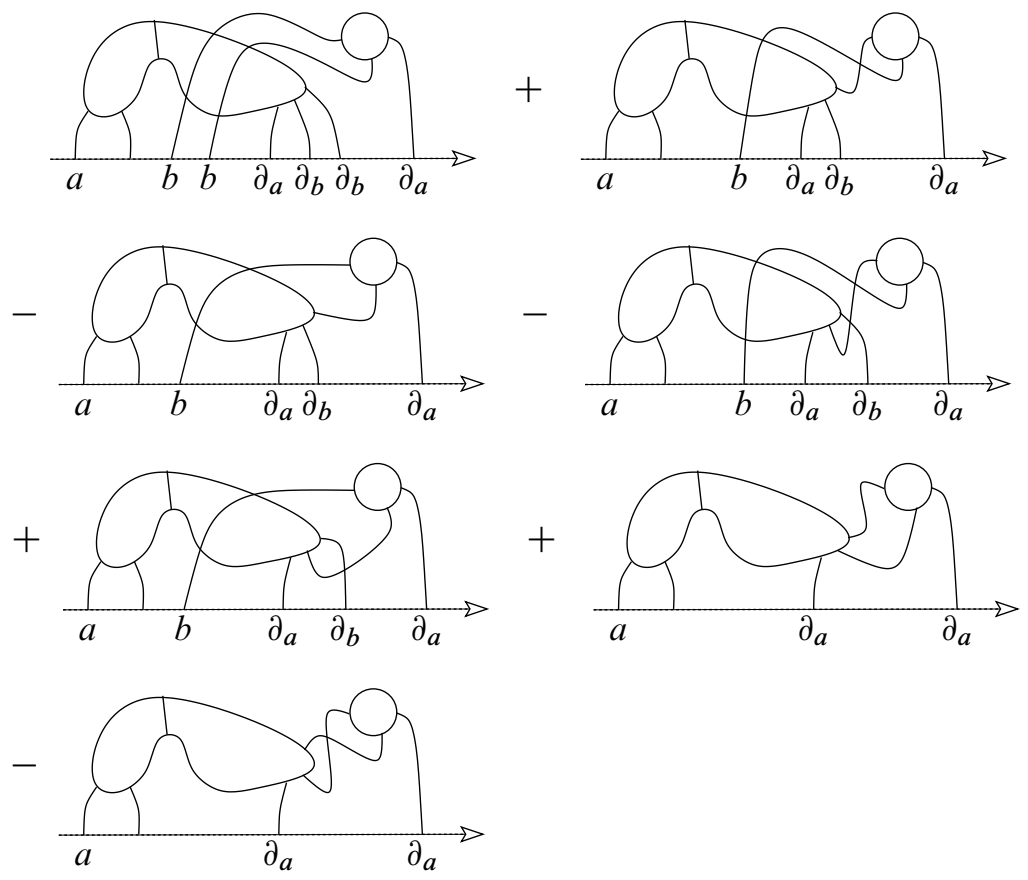

Figure 7: The final result of the diagram operation started in Figure 6

Definition 3.2.5 Let $v$ and $w$ be power series from $\widehat{\mathcal{W}}_{\mathbf{F}} \llbracket a, b, \partial_{a}, \partial_{b} \rrbracket$. If for every pair $(i, j) \in \mathbb{N}_{0} \times \mathbb{N}_{0}$ it is true that

$$
\operatorname{pr}^{(i, j)}\left(v^{(k, l)} \vdash w^{(m, n)}\right)=0
$$

for all but finitely many pairs $((k, l),(m, n))$, then we say that the product $v \vdash w$ is convergent, in which case it is defined to be

$$
\sum_{(k, l),(m, n) \in \mathbb{N}_{0} \times \mathbb{N}_{0}}\left(v^{(k, l)} \vdash w^{(m, n)}\right) \in \widehat{\mathcal{W}}_{\mathbf{F}} \llbracket a, b, \partial_{a}, \partial_{b} \rrbracket .
$$

\subsection{A convenient graphical method for doing diagram operations}

In certain of the computations to come later in this paper, we'll need to be able to give a direct construction of all the terms that contribute to a diagram operation, all at once, together with an easy way to determine the signs of those contributions. For this purpose we'll now introduce a convenient visual method for doing a diagram operation.

Definition 3.3.1 Consider two operator Weil diagrams $v$ and $w$. Let $\mathrm{Op}(v)$ denote the set of operator legs of $v$, and let $\operatorname{Par}(w)$ denote the set of parameter legs of $w$. 
A gluing of $v$ onto $w$ is an injection of a (possibly empty) subset of $\operatorname{Op}(v)$ into $\operatorname{Par}(w)$ that respects labels (so a $\partial_{a}$-labelled leg of $v$ will only be mapped to an $a$-labelled leg of $w$, etc). Let $\mathcal{G}(v, w)$ denote the set of gluings of $v$ onto $w$.

Below we'll explain how to associate a term $t(v, w, \sigma)$ to each gluing $\sigma \in \mathcal{G}(v, w)$, and then we'll define the operation of $v$ on $w$ to be: $v \vdash w=\sum_{\sigma \in \mathcal{G}(v, w)} t(v, w, \sigma)$. This construction will obviously agree with the first definition of the operation that we gave in Section 3.2.1.

In the discussion to follow, we'll consider the example of the diagrams
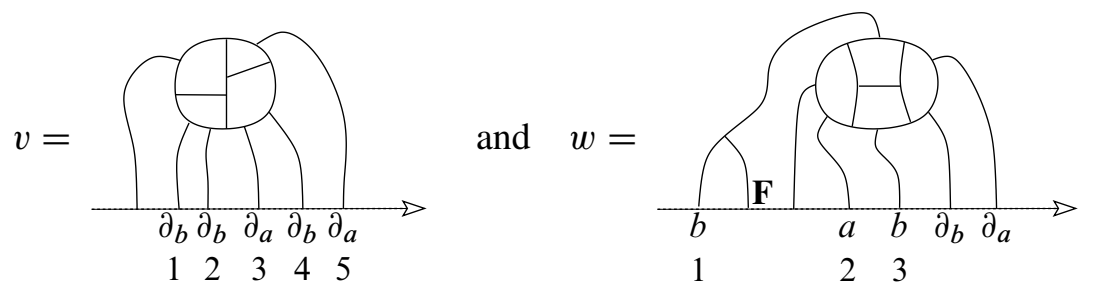

For the purposes of the discussion, the operator legs of $v$ have been numbered from left to right, and so have the parameter legs of $w$, as displayed by the above diagrams. Below, we'll construct the term

$$
t\left(v, w,\left(\begin{array}{lll}
2 & 4 & 5 \\
1 & 3 & 2
\end{array}\right)\right) .
$$

The term $t(v, w, \sigma)$ corresponding to some gluing $\sigma \in \mathcal{G}(v, w)$ will be constructed by the following procedure. To begin, place the operator legs of $v$ up the left-hand side of a grid, and the nonoperator legs of $w$ across the top of the grid, above an orienting line, as shown in Figure 8.

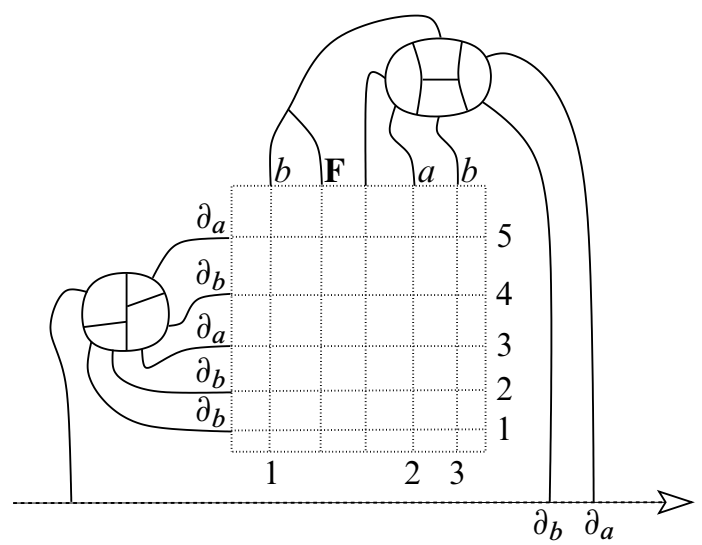

Figure 8: Arrange the diagrams around a grid 
Next, join up legs along the grid according to the gluing. Join up grade 1 legs using a full line, and grade 2 legs using a dashed line (this is so that we will be able to easily read off the sign of the term at the end of the construction). To finish: carry any remaining legs on the top of the grid to the orienting line. For grade 1 legs use a full line and for grade 2 legs use a dashed line. And carry any remaining operator legs lying along the left-hand side of the grid to the right-hand side of the grid, and then place them on the orienting line using nested right-angles. The result in this example is the diagram displayed in Figure 9.

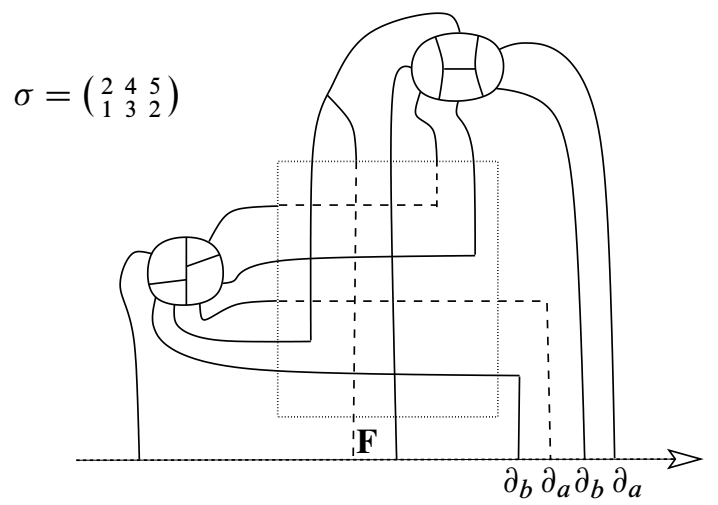

Figure 9: The gluing arising from the injection $\sigma$

Let $x$ denote the number of intersections between full lines displayed within the box. The term $t(v, w, \sigma)$ is just the diagram that has been constructed (with the dashed lines now filled in), with a sign $(-1)^{x}$ out the front.

\subsection{Associativity}

It is an exercise in this graphical method to show that the operation product is associative:

Proposition 3.4.1 Let $u, v$ and $w$ denote operator Weil diagrams. Then

$$
u \vdash(v \vdash w)=(u \vdash v) \vdash w .
$$

There is a nice way to organize the terms contributing to these triple-products. To illustrate this, consider the case of
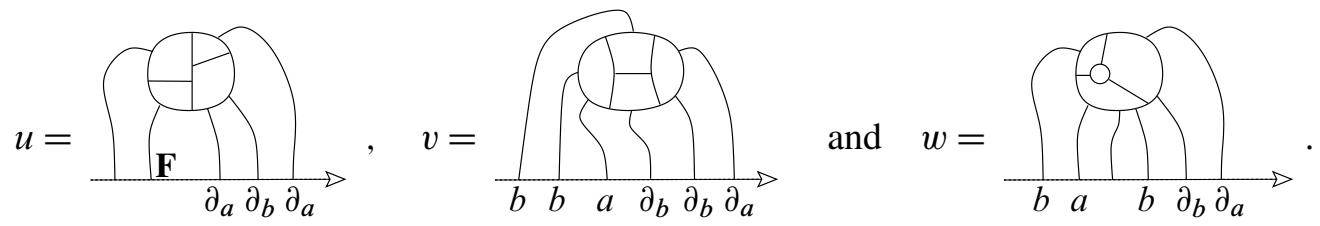
We can assemble the three diagrams around the edges of a "step-ladder" grid, as shown in Figure 10. To prove associativity you should:

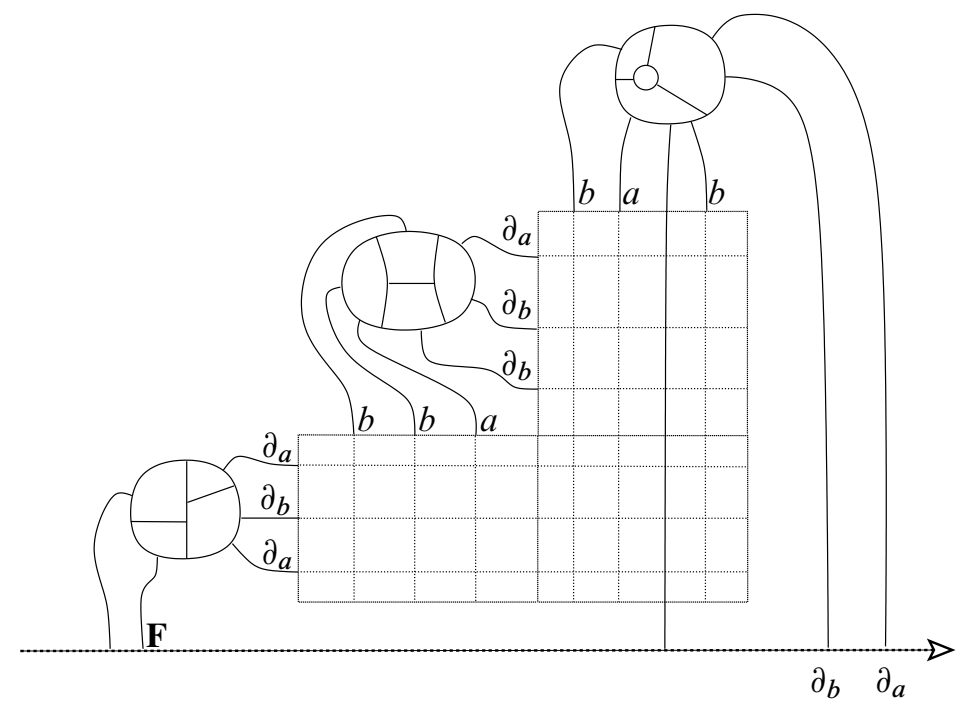

Figure 10: The diagram you use to prove associativity

- Observe how to express $u \vdash(v \vdash w)$ as a sum of diagrams built from this step-ladder grid.

- Observe how to express $(u \vdash v) \vdash w$ in the same way.

- Observe that the terms of the two sums correspond.

A detailed proof appeared in the first version of this paper [10].

3.4.2 Associativity and power series We recommend this section for the second reading; it consists of some unsurprising details about associativity and convergence.

In the computations that are the core of this work, we'll need to re-bracket certain products of power series. We've just shown that we can re-bracket products of the generators; to re-bracket products of power series proves to be a more delicate affair (because of convergence issues). To avoid getting bogged down by the logic of our definitions, we'll introduce a simple finiteness condition (Condition (§), below). When Condition (§) holds, for a triple $u, v$ and $w$ of power series from $\widehat{\mathcal{W}}_{\mathbf{F}} \llbracket a, b, \partial_{a}, \partial_{b} \rrbracket$, then it will be true that

$$
(u \vdash v) \vdash w=u \vdash(v \vdash w) .
$$

We'll state the condition as a lemma: 
Lemma 3.4.3 Let $u, v$ and $w$ be power series from $\widehat{\mathcal{W}}_{\mathbf{F}} \llbracket a, b, \partial_{a}, \partial_{b} \rrbracket$. Assume that:

- The product $u \vdash v$ converges.

- The product $v \vdash w$ converges.

- For all $(i, j)$, there are only finitely many triples $((k, l),(m, n),(p, q))$ with the property that

$$
\operatorname{pr}^{(i, j)}\left(\left(u^{(k, l)} \vdash v^{(m, n)}\right) \vdash w^{(p, q)}\right) \neq 0 .
$$

Then the products $(u \vdash v) \vdash w$ and $u \vdash(v \vdash w)$ converge, and

$$
(u \vdash v) \vdash w=u \vdash(v \vdash w) .
$$

There is also a version for the other bracketting. It will be a straightforward matter to check that this condition holds in any situation that we perform a re-bracketting.

Proof First, note that $(u \vdash v) \vdash w$ obviously converges. (Otherwise Condition (§) would be violated.) Second, note that because we can re-bracket the generators, Condition $(\S)$ implies its re-bracketted version: that for each $(i, j)$ there are only finitely many triples $((k, l),(m, n),(p, q))$ such that

$$
\operatorname{pr}^{(i, j)}\left(u^{(k, l)} \vdash\left(v^{(m, n)} \vdash w^{(p, q)}\right)\right) \neq 0 .
$$

Thus $u \vdash(v \vdash w)$ converges as well. Thirdly, note that Condition (§) implies that the expression

$$
\sum_{(k, l),(m, n),(p, q)}\left(u^{(k, l)} \vdash v^{(m, n)}\right) \vdash w^{(p, q)}
$$

makes sense. It is almost tautological that it is equal to $(u \vdash v) \vdash w$. And similarly, $\left(\S^{\prime}\right)$ implies that

$$
u \vdash(v \vdash w)=\sum_{(k, l),(m, n),(p, q)} u^{(k, l)} \vdash\left(v^{(m, n)} \vdash w^{(p, q)}\right) .
$$

Associativity of products of generators gives the required equality.

The major reason we need such detail is that there are products $(u \vdash v) \vdash w$ which don't satisfy Condition ( $\S$ ) but which nevertheless converge. This makes general statements difficult. 


\section{Expressing the composition as an operator product}

\subsection{How the proof of the main theorem is structured}

The computation that is the subject of Main Theorem is based on an expression of the value of the composition

$$
\mathcal{B} \stackrel{\Upsilon}{\longrightarrow} \mathcal{W} \stackrel{\chi \mathcal{W}}{\longrightarrow} \widetilde{\mathcal{W}} \stackrel{\pi}{\longrightarrow} \widehat{\mathcal{W}} \stackrel{B_{\bullet} \rightarrow \mathbf{F}}{\longrightarrow} \widehat{\mathcal{W}}_{\mathbf{F}} \stackrel{\lambda}{\longrightarrow} \widehat{\mathcal{W}}_{\wedge}
$$

on some symmetric Jacobi diagram in terms of the operation product $\vdash$.

The expression uses a linear map $\mathrm{B}_{l \mapsto \partial_{a}}: \mathcal{B} \rightarrow \widehat{\mathcal{W}}_{\wedge} \llbracket a, b, \partial_{a}, \partial_{b} \rrbracket$ which acts on a Jacobi diagram $v$ by first choosing an ordering of the legs of $v$ and then labelling every leg with a $\partial_{a}$. For example,

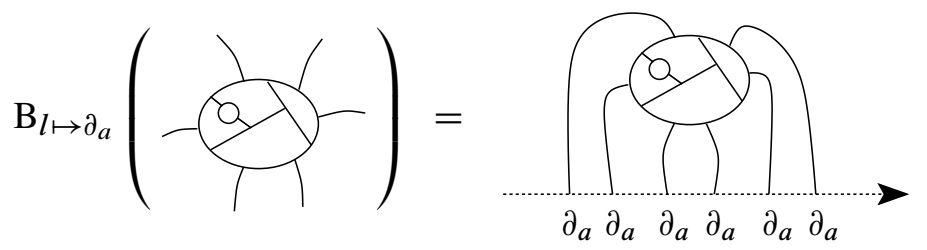

Theorem 4.1.1 Let $v$ be an element of $\mathcal{B}$, the space of symmetric Jacobi diagrams. Then the element $\left(\lambda \circ B_{\bullet \rightarrow \mathrm{F}} \circ \pi \circ \chi_{\mathcal{W}} \circ \Upsilon\right)(v)$ is equal to

$$
\left[B_{l \mapsto \partial_{a}}(v) \vdash\left(\exp _{\#}\left(\bigcap_{a} \mathbf{F}_{\triangle \rightarrow}\right) \# \mathcal{X}\right)\right]_{a, b, \partial_{a}, \partial_{b}=0}
$$

where $\mathcal{X}$ is equal to

$$
\exp _{\vdash}\left(-\frac{1}{2} \quad \bigcap_{a} \lambda_{\partial_{b} \partial_{b}}>\right) \vdash \lambda\left(\exp _{\#}\left(\frac{1}{2} \bigcap_{a} \lambda_{\rightarrow \rightarrow}+\bigcap_{b}\right)\right) .
$$

This section is devoted to the proof of this theorem. In the next section, Section 5, we commence the computation of the $b, \partial_{b}=0$ piece of $\mathcal{X}$ by performing the $\lambda$ operation in the second factor above. (Notice that computation of this piece of $\mathcal{X}$ is sufficient because we immediately set $b$ and $\partial_{b}$ to zero.) Section 6 takes that result and performs the remaining operation product, while setting $b, \partial_{b}=0$.

To state the final computation of $[\mathcal{X}]_{b, \partial_{b}=0}$ we'll employ the following notation for the $a$-labelled legs. Note that the $a$-labelled legs commute with every type of leg, and so can be moved around freely. It proves clearer to avoid drawing them in explicitly. We'll record the $a$-labelled legs by (locally) orienting the edge they are incident to, and labelling that edge with the appropriate power series in $a$. 
Section 6 completes the computation that:

\section{Theorem 4.1.2}

$$
[\mathcal{X}]_{b, \partial_{b}=0}=\exp _{\#}\left(\bigcirc_{-}^{\frac{1}{2} \ln \left(\frac{\sinh a / 2}{a / 2}\right)}\right) .
$$

Substituting this computation into Theorem 4.1.1 completes the proof of the main theorem, Main Theorem. It may take a few minutes of doodling to agree with this claim. The point is that when you apply $B_{l \mapsto \partial_{a}}(v)$ to the $\exp (\cdot) \# \mathcal{X}$ term, followed by setting all parameters to zero, then every leg of $v$ either becomes an $\mathbf{F}-\mathrm{leg}$, or is attached to a leg of $\mathcal{X}$, in "all possible ways". The factorial appearing in the denominator (arising from the exponential) exactly matches the factorial appearing in the denominator of the averaging map $\chi_{\mathcal{B}}$.

\subsection{The organization of this section}

The remainder of this section is devoted to the proof of the expression presented above in Theorem 4.1.1. We'll build up to the theorem piece-by-piece. The sequence of steps is as follows.

- Section 4.3 focuses on the following piece of the expression:

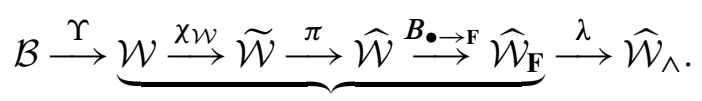

This part contains the most important idea, that you can graded-average a diagram by applying a corresponding formal differential operator to a formal exponential.

- Then, in Section 4.4, we derive an operator expression for the first step in the composition, the "hair-splitting map":

$$
\underbrace{\mathcal{B} \stackrel{\Upsilon}{\longrightarrow} \mathcal{W}} \stackrel{\chi \mathcal{W}}{\longrightarrow} \widetilde{\mathcal{W}} \stackrel{\pi}{\longrightarrow} \widehat{\mathcal{W}} \stackrel{B_{\bullet} \rightarrow \mathbf{F}}{\longrightarrow} \widehat{\mathcal{W}}_{\mathbf{F}} \stackrel{\lambda}{\longrightarrow} \widehat{\mathcal{W}}_{\wedge} .
$$

- In Section 4.5 we immediately insert the expression we just derived for hairsplitting into the expression developed in Section 4.3, and do some simple rearrangements, to obtain a formula for most of the composition:

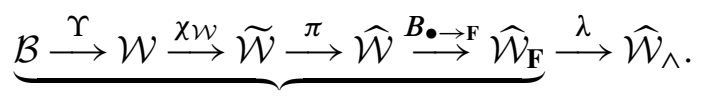

- The final operation in the composition is to apply $\lambda$. In Section 4.6 we explain that $\lambda$ can be commuted through the operator expression so that the first step in the construction is $\lambda$ operating on the formal exponential. That completes the development of the expression in Theorem 4.1.1. 


\subsection{Using operator diagrams to average}

We begin by developing an expression for the graded-averaging map.

Definition 4.3.1 Define a linear map

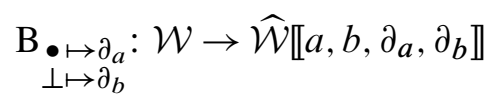

by replacing legs according to the rules

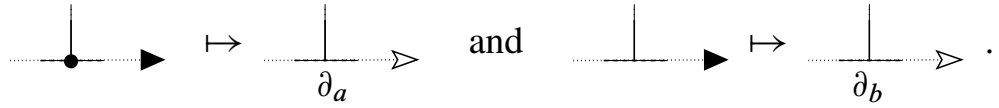

The key proposition is the following one, which says that we can take the gradedaverage of a diagram by changing it into an operator, applying the resulting operator to an exponential of formal parameters, then setting all parameters to zero. The map which sets all the parameters to zero, denoted $[.]_{A, b}, \partial_{a}, \partial_{b}=0$ below, is precisely $\mathrm{pr}^{(0,0)}$, the projection of the $(0,0)$ factor out of the power series.

Proposition 4.3.2 Let $v \in \mathcal{W}$. Then

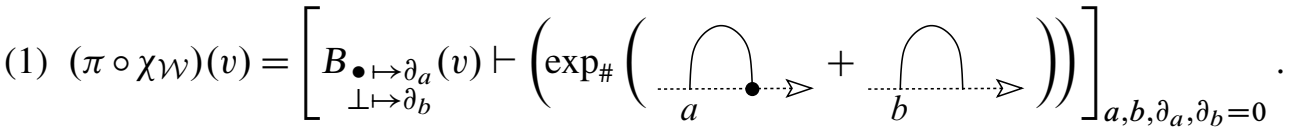

Proof Both sides are linear maps, so it suffices to check this formula on generators. So take some symmetric Weil diagram $v$, and assume that it has $p$ grade 2 legs and $q$ grade 1 legs. We'll evaluate the value that the right-hand side takes on $v$ and observe that it is precisely the signed average of $v$, as required.

For convenience, write

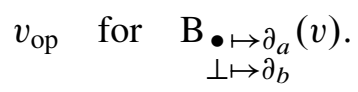

Consider, then, the exponential that $v_{\text {op }}$ is to be applied to. We'll index its terms by the set of words (including the empty word) that can be built from the symbols $A$ and $B$. Given some such word $w$, let $f_{w}$ denote the corresponding diagram. For example,

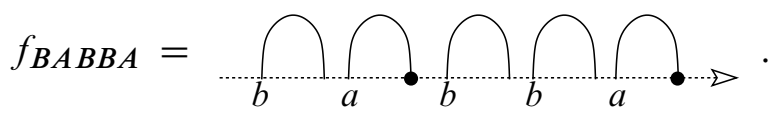

We can expand the right-hand-side of Equation (1) to get

$$
\frac{1}{(p+q) !} \sum_{\begin{array}{c}
\text { words } w \text { built } \\
\text { from } p \text { copies of } A \\
\text { and } q \text { copies of } B .
\end{array}}\left[v_{\mathrm{op}} \vdash f_{w}\right]_{a, b, \partial_{a}, \partial_{b}=0} .
$$


Fix such a word $w$ and let's proceed to calculate $\left[v_{\mathrm{op}} \vdash f_{w}\right]_{a, b, \partial_{a}, \partial_{b}=0}$. For example, if

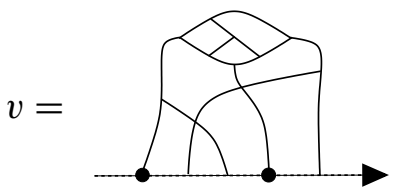

and $w=B A B B A$, then we wish to calculate
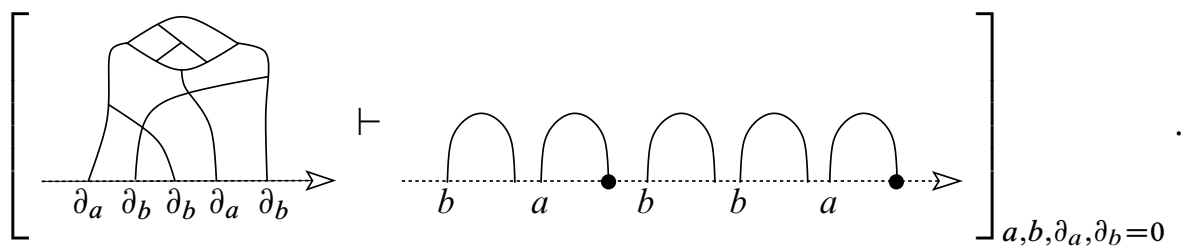

To do such a computation directly, it helps to employ the graphical method for doing diagram operations that was described in Section 3.3.

Let's take a moment to recall this method. We begin by placing the operator legs of $v_{\text {op }}$ up the left-hand side of a grid, and the legs of $f_{w}$ along the top of the grid. In the case of this example, the diagram we draw is exhibited in Figure 11. According to

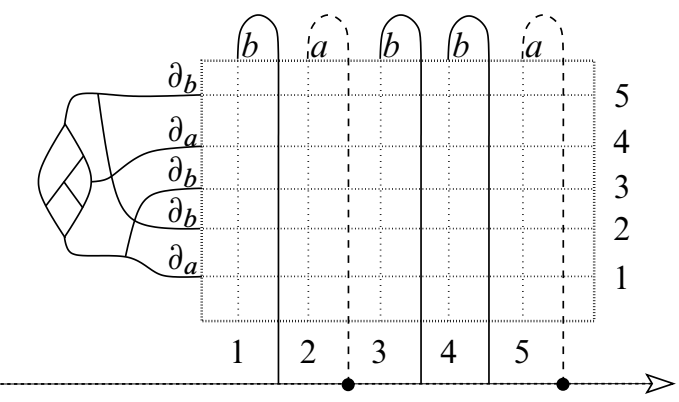

Figure 11: The diagram which organizes the computation of the example

this method, we get precisely one contribution $t\left(v_{\mathrm{op}}, f_{w}, \sigma\right)$ to the operation for every permutation $\sigma:\{1,2,3,4,5\} \rightarrow\{1,2,3,4,5\}$ which respects the parameters. (To be precise, these are the contributions that will survive after the parameters get sent to zero at the end of the operation.) So if we let Perm ${ }_{n}$ denote the set of all permutations on $\{1, \ldots, n\}$ and let $\operatorname{Perm}_{p+q}(v, w) \subset \operatorname{Perm}_{p+q}$ denote the set of permutations respecting the parameters, then we can write

$$
\left[v_{\mathrm{op}} \vdash f_{w}\right]_{a, b, \partial_{a}, \partial_{b}=0}=\sum_{\sigma \in \operatorname{Perm}_{p+q}(v, w)} t\left(v_{\mathrm{op}}, f_{w}, \sigma\right),
$$


where $t\left(v_{\mathrm{op}}, f_{w}, \sigma\right)$ is determined by the usual graphical method. Recall that the sign

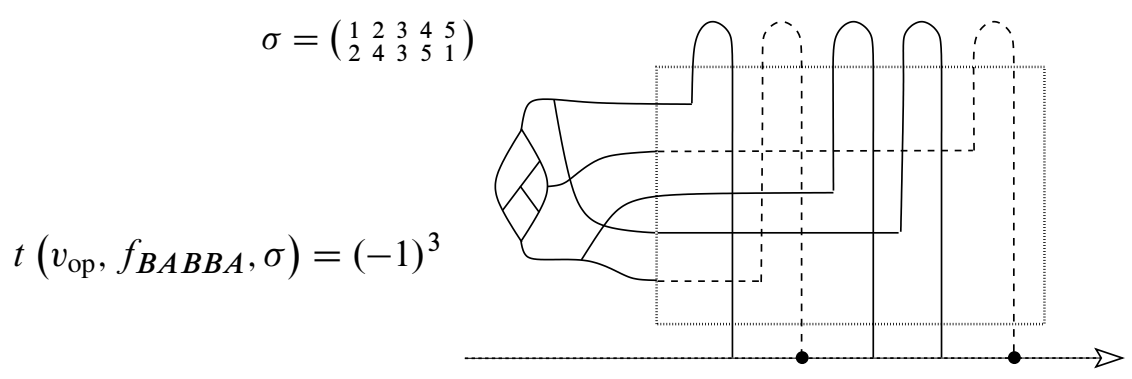

Figure 12: The contribution corresponding to $\sigma$

of the contribution is determined by counting the number of intersections between full lines displayed within the box. For example, the contribution corresponding to the permutation

$$
\left(\begin{array}{lllll}
1 & 2 & 3 & 4 & 5 \\
2 & 4 & 3 & 5 & 1
\end{array}\right)
$$

is displayed in Figure 12.

Here is what we need to notice: the diagram $t\left(v_{\mathrm{op}}, f_{w}, \sigma\right)$ put out by this operation is (up to some to-be-determined sign) precisely what we get by permuting the legs of the original diagram using the permutation $\sigma$. To state this observation precisely: Given a permutation $\sigma \in \operatorname{Perm}_{p+q}$, let $v^{\sigma}$ denote the diagram one gets by permuting (without introducing signs) the legs of $v$ according to $\sigma$.

The required observation is:

Lemma 4.3.3 Let $v$ be some diagram from $\mathcal{W}$ with $p$ grade 2 legs and $q$ grade 1 legs. Let $w$ be some word which uses $p$ copies of the symbol $A$ and $q$ copies of the symbol $B$. Let $\sigma$ be some permutation from $\operatorname{Perm}_{p+q}(v, w)$. Then

$$
t\left(v_{\mathrm{op}}, f_{w}, \sigma\right)=\varphi(\sigma) v^{\sigma},
$$

where $\varphi(\sigma)$ is the product of a $(-1)$ for every pair of grade 1 legs of $v$ which reverse their order in $v^{\sigma}$.

We'll leave the reader to see that this lemma is true. A detailed proof appeared in the first version of this paper [10]. 
Now we can put it all together. According to Equation (2) and Equation (3), the right-hand side of the Equation (1) can be written

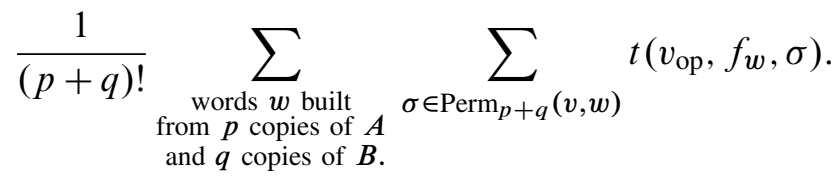

Substituting the result of the lemma into this, we get

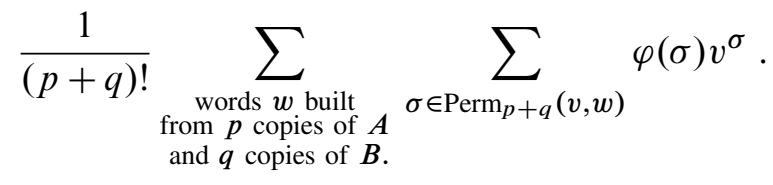

This is just $\pi \circ \chi_{\mathcal{W}}$, the required graded averaging map.

One step extends this proposition to give:

Corollary 4.3.4 Let $v \in \mathcal{W}$. Then $\left(B_{\bullet \rightarrow \mathrm{F}} \circ \pi \circ \chi_{\mathcal{W}}\right)(v)$ is given by the expression

$$
\left[\underset{\substack{\bullet \mapsto \partial_{a} \\ \perp \mapsto \partial_{b}}}{B}(v) \vdash\left(\exp _{\#}\left(\bigcap_{a} \mathbf{F}_{\supset}+\frac{1}{2} \bigcap_{a}+\bigcap_{b}+\bigcap_{b}\right)\right)\right]_{a, b, \partial_{a}, \partial_{b}=0} .
$$

\subsection{Hair-splitting with operator diagrams}

We now turn our focus to the hair-splitting map.

Proposition 4.4.1 Let $v \in \mathcal{B}$. Then the equation

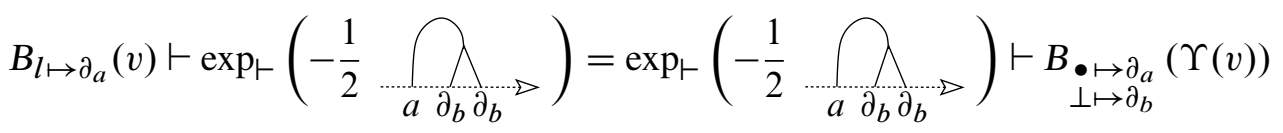

holds in $\widehat{\mathcal{W}}_{\mathbf{F}} \llbracket a, b, \partial_{a}, \partial_{b} \rrbracket$.

Proof As the two sides of this equation are both linear maps, it suffices to show that the equation holds for generators of $\mathcal{B}$. So let $v$ be a symmetric Jacobi diagram. We begin by expanding the left-hand side of Proposition 4.4.1 in the following way:

$$
\sum_{n=0}^{\infty}\left(\left(-\frac{1}{2}\right)^{n} \frac{1}{n !}\right) B_{l \mapsto \partial_{a}}(v) \vdash(\overbrace{\overbrace{a \partial_{b} \partial_{b}} \cdots \cdots \cdots \widehat{\lambda}_{a \partial_{b} \partial_{b}}}^{n}) .
$$


Now consider the diagram operation in the above sum. Recalling how to do diagram operations (see Section 3.3), we get (letting $\mathcal{L}$ denote the set of the legs of $v$ )

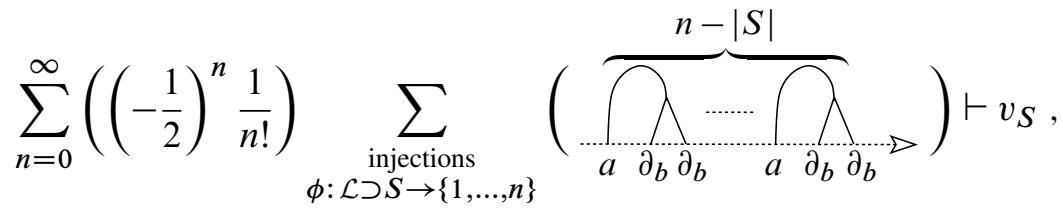

where, for example,

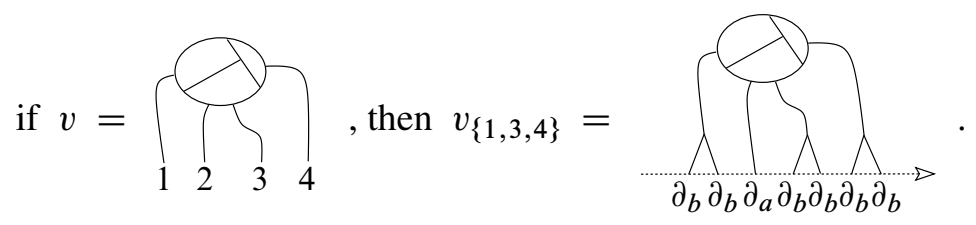

Now for every subset $S \subset \mathcal{L}$ of legs there are $n ! /(n-|S|)$ ! injections $\phi: S \rightarrow$ $\{1, \ldots, n\}$. Thus the above expression may be rewritten

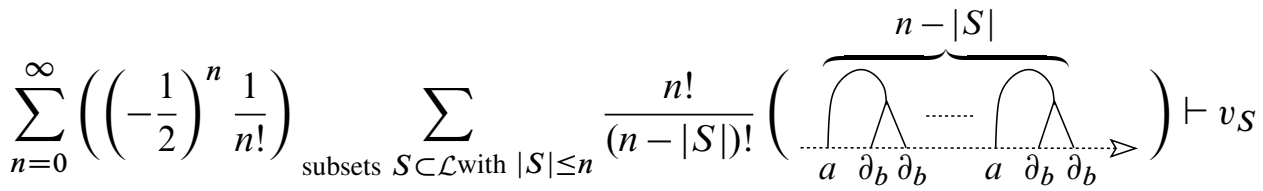

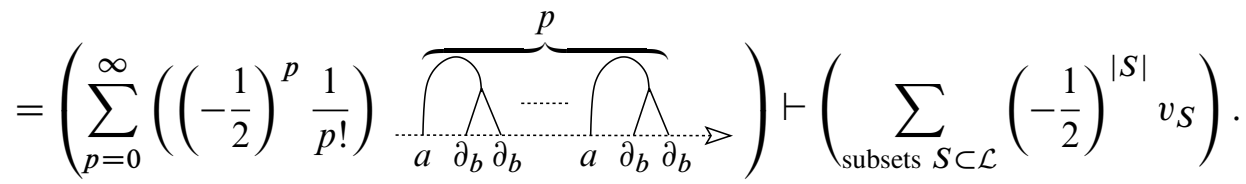

This is the right hand side of Proposition 4.4.1.

\subsection{Putting the pieces together}

Let $v$ be an element of $\mathcal{B}$. If we substitute $\Upsilon(v)$ directly into Corollary 4.3.4 then we are given the following expression for $\left(\mathrm{B}_{\bullet \rightarrow \mathrm{F}} \circ \pi \circ \chi_{\mathcal{W}} \circ \Upsilon\right)(v)$ :

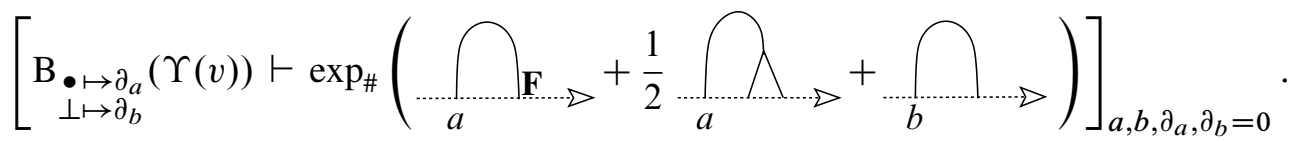

We now wish to use Proposition 4.4.1 to re-express this as a direct function of $v$. To this end, we begin by inserting the missing piece of that proposition into the front of 
this expression, giving

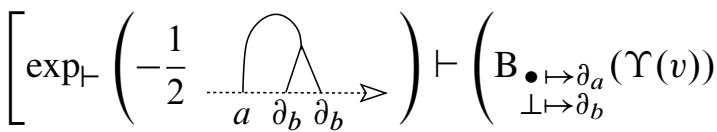

$$
\begin{aligned}
& \left.\left.\vdash \exp _{\#}\left(\bigcap_{a} \mathbf{F}_{\triangle}+\frac{1}{2} \bigcap_{a} \hat{⿴}_{\rightarrow}+\bigcap_{b}\right)\right)\right]_{a, b, \partial_{a}, \partial_{b}=0} .
\end{aligned}
$$

We can do this because all operation products in the resulting expression converge and because, when we set the parameter $a$ to zero, all the introduced terms will vanish.

Then we perform an associativity rearrangement (an ultra-careful reader may wish to read Lemma 3.4.3 and then check that Condition ( $($ ) holds before rearranging). Then we apply Proposition 4.4.1 to replace $\Upsilon$. And finally we do another associativity rearrangement to get

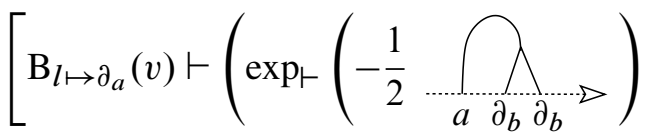

$$
\begin{aligned}
& \left.\left.\vdash \exp _{\#}\left(\bigcap_{a} \mathbf{F}_{\triangle}+\frac{1}{2} \bigcap_{a} \bigcap_{\rightarrow}+\bigcap_{b}\right)\right)\right]_{a, b, \partial_{a}, \partial_{b}=0} .
\end{aligned}
$$

Finally, observe that $a$-labelled legs commute with all other types of legs, and F-legs commute with all other legs (except other F-legs), so some straightforward rearrangements allow us to write (using \{\} instead of () only to make this equation easier on the eye)

$$
\begin{aligned}
& {\left[\mathrm { B } _ { l \mapsto \partial _ { a } } ( v ) \vdash \left\{\exp _{\#}\left(\bigcap_{a} \mathbf{F}_{\supset \rightarrow}\right) \#\right.\right.}
\end{aligned}
$$

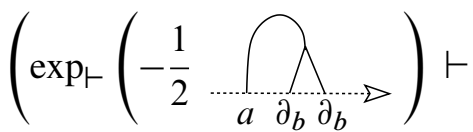

$$
\begin{aligned}
& \left.\left.\left.\exp _{\#}\left(\frac{1}{2} \bigcap_{a} \bigwedge_{\triangle \rightarrow}+\bigcap_{b}\right)\right)\right\}\right]_{a, b, \partial_{a}, \partial_{b}=0} .
\end{aligned}
$$

\subsection{Commuting $\lambda$ through the expression}

The remaining step in the development of Theorem 4.1.1 is to apply $\lambda$ to the expression (4). In this section we'll observe that we can commute $\lambda$ through the expression so that it operates directly on the right-most exponential. 
For the purposes of this discussion we'll refer to legs of the form

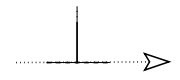

as $\perp-$ legs.

In Section 1.11 we recalled that $\lambda$ is a map $\lambda: \widehat{\mathcal{W}}_{\mathbf{F}} \rightarrow \widehat{\mathcal{W}}_{\wedge}$ basically defined by "gluing $\perp$-legs together in all possible ways (with coefficients)". In this section we'll extend $\lambda$ to the situation where there are some parameter and operator legs present, to $\lambda: \widehat{\mathcal{W}}_{\mathbf{F}} \llbracket a, b, \partial_{a}, \partial_{b} \rrbracket \rightarrow \widehat{\mathcal{W}}_{\wedge} \llbracket a, b, \partial_{a}, \partial_{b} \rrbracket$. The purpose of this section is to prove the following proposition.

Proposition 4.6.1 Let $v$ and $w$ be elements of $\widehat{\mathcal{W}}_{\mathbf{F}} \llbracket a, b, \partial_{a}, \partial_{b} \rrbracket$ such that the product $v \vdash w$ converges, and assume that $v$ can be expressed without $\perp$-legs. Then

$$
\lambda(v \vdash w)=v \vdash \lambda(w) .
$$

Before turning to the proof, let's repeat the definition of $\lambda$ (in this more general context). Consider some diagram $w$, a generator of $\widehat{\mathcal{W}}_{\mathbf{F}} \llbracket a, b, \partial_{a}, \partial_{b} \rrbracket$. Let $\mathcal{L}_{\perp}(w)$ denote the set of $\perp$-legs of $w$. Recall that a pairing of $w$ is a (possibly empty) set of disjoint 2-element subsets of $\mathcal{L}_{\perp}$. As before, $\mathcal{P}(w)$ denotes the set of pairings of $w$. We then define $\lambda$ by $\lambda(w)=\sum_{\wp \in \mathcal{P}(w)} \mathcal{D}_{\wp}(w)$ where the term $\mathcal{D}_{\wp}(w)$ is constructed by the graphical procedure described in Section 1.11. The only new detail that can occur in this more general context is that when computing the sign of the contribution coming from some pairing, one should pay attention also to the grades of the parameter legs. For example, if

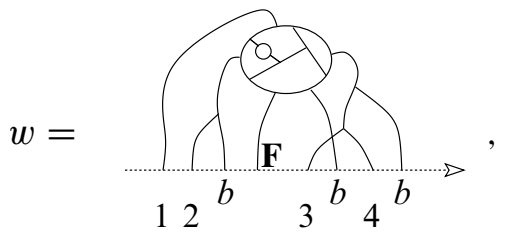

then

$$
\mathcal{D}_{\{\{1,4\},\{2,3\}\}}(w)=(-1)^{3}\left(\frac{1}{2}\right)^{2}
$$

Proof It suffices to show that

$$
\lambda(v \vdash w)=v \vdash \lambda(w)
$$

is true for generators. 
So let $v$ and $w$ be operator Weil diagrams and assume that $v$ has no $\perp-$ legs. It follows from the construction of the operations $\lambda$ and $\vdash$ that the two sides of Equation (5) are sums indexed by the same set. Define that set $\mathcal{P G}$ to consist of pairs $(\wp, \sigma)$, where $\wp$ is a pairing $\wp \in \mathcal{P}(w)$ and $\sigma$ is a gluing $\sigma \in \mathcal{G}(v, w)$. Then:

(1) $\lambda(v \vdash w)=\sum_{(\wp, \sigma) \in \mathcal{P} \mathcal{G}} \mathcal{D}_{\wp}(t(v, w, \sigma))$,

(2) $v \vdash \lambda(w)=\sum_{(\wp, \sigma) \in \mathcal{P G}} t\left(v, \mathcal{D}_{\wp}(w), \sigma\right)$,

where $t(v, w, \sigma)$ is the notation used in Section 3.3 for the term that arises when $v$ is applied to $w$ using the gluing $\sigma$. The required equality is established by showing that for every pair $(\wp, \sigma) \in \mathcal{P G}$,

$$
\mathcal{D}_{\wp}(t(v, w, \sigma))=t\left(v, \mathcal{D}_{\wp}(w), \sigma\right) .
$$

We'll leave it as an exercise for the reader to understand, using the graphical constructions of the two sides of the equation, why this equality holds. A simple example should reveal the truth of the matter. A detailed discussion of this equality appears in the first version of this paper [10].

\section{Computing the operator product I: The inner-most piece}

The remainder of this paper is occupied with the computation of the key term $\mathcal{X}$ appearing in Theorem 4.1.1. We begin with the inner-most piece. The objective of this section is to prove the following theorem.

Theorem 5.0.2 In $\widehat{\mathcal{W}}_{\wedge} \llbracket a, b, \partial_{a}, \partial_{b} \rrbracket$ the expression

is equal to
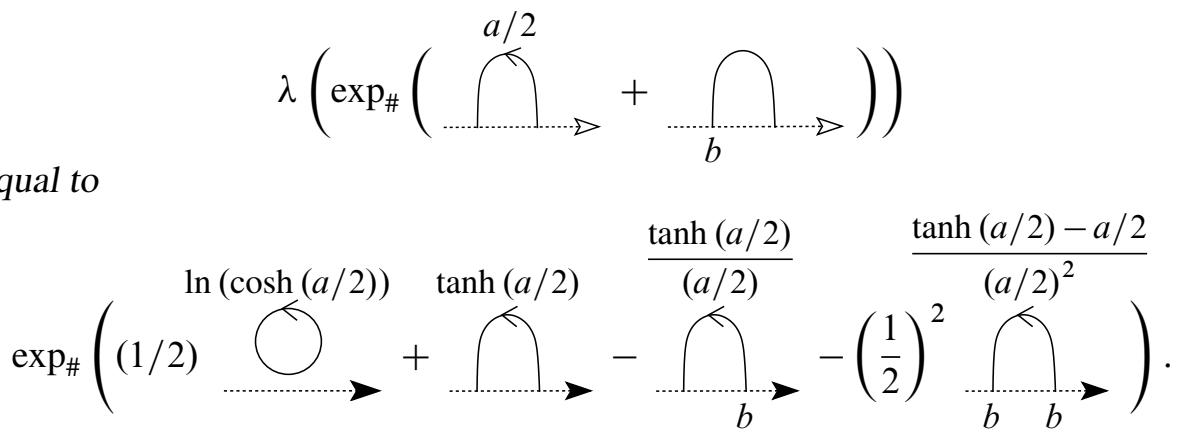

This section consists of two subsections. In Section 5.1 we show that the left-hand side of the above equation can be expressed as an exponential of the series of terms with connected diagrams that arise from the evaluation of $\lambda$. In Section 5.2 we'll perform a detailed calculation of that series. 


\subsection{An exponential of connected diagrams}

Consider the terms that arise when you compute the first of the two expressions above. We'll index these terms with a certain set $\mathcal{T}$. The set $\mathcal{T}$ is defined to be the set of pairs $(w, \wp)$ consisting of a nonempty word $w$ in the symbols $A$ and $B$ and a (possibly empty) family $\wp$ of disjoint 2 -element subsets of the set $\{1,2,3, \ldots, 2 \# A+\# B\}$ (where $\# A$ and $\# B$ denote the number of appearances in the word $w$ of the symbols $A$ and $B$ respectively). If $\tau=(w, \wp)$ we'll often write $|\tau|$ for $|w|$, the length of the word $w$.

Given such a pair $(w, \wp)$, the corresponding term $T_{(w, \wp)}$ is constructed in two steps. The first step is to replace each appearance in the word $w$ of

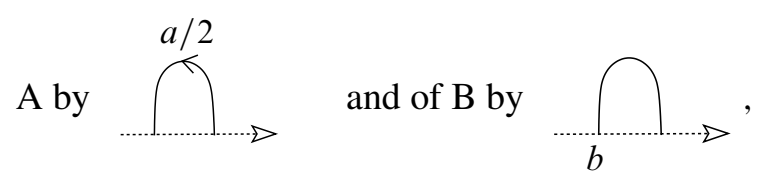

so as to construct a single diagram along an orienting line in the order dictated by $w$. The second step is to pair up (with appropriate coefficients) the $\perp$-legs of this diagram according to the pairing §. Some examples appearing shortly in Section 5.1.2 should make this clear.

With this notation the main expression can be expanded as follows:

$$
\lambda\left(\exp _{\#}\left(\bigcap_{b}^{a / 2}+\bigcap_{\rightarrow \rightarrow \infty}\right)\right)=1+\sum_{\tau \in \mathcal{T}} \frac{1}{|\tau| !} T_{\tau} .
$$

Our first task is to show that the right-hand-side of this equation can be organized into an exponential of connected diagrams. To be precise: let $\mathcal{T}_{\mathcal{C}} \subset \mathcal{T}$ denote the subset consisting of those pairs $(w, \wp)$ whose corresponding term $T_{(w, \wp)}$ is connected. (By connected we mean that the graph is connected when the orienting line is ignored.) Then:

Theorem 5.1.1 The following equation holds in $\widehat{\mathcal{W}}_{\wedge} \llbracket a, b, \partial_{a}, \partial_{b} \rrbracket$ :

$$
\lambda\left(\exp _{\#}\left(\bigcap_{b}^{a / 2}+\bigcap_{b \rightarrow \infty}\right)\right)=\exp _{\#}\left(\sum_{\tau \in \mathcal{T}_{\mathcal{C}}} \frac{1}{|\tau| !} T_{\tau}\right) .
$$

We'll build up to this theorem with a number of combinatorial lemmas. The direct computation of $\sum_{\tau \in \mathcal{T}_{\mathcal{C}}}(1 /|\tau| !) T_{\tau}$ is taken up in Section 5.2. 
5.1.2 The content of a pairing Consider some pair $(w, \wp)$. The corresponding diagram $T_{(w, \wp)}$ decomposes into a number a connected components. To each connected component $x$ we can associate some other pair $\left(w_{x}, \wp_{x}\right)$. For example, consider the diagram corresponding to $(A A B A A A,\{\{2,5\},\{4,6\},\{3,9\},\{7,11\},\{8,10\}\})$. It is

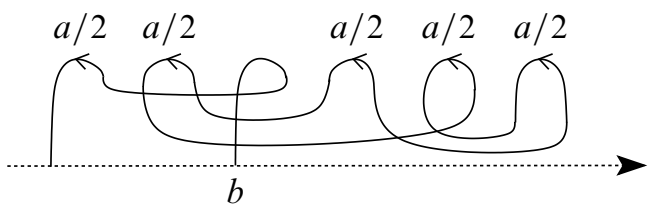

This diagram has 2 connected components. For example, one component corresponds to the pair $(A A A A,\{\{1,6\},\{2,3\},\{4,8\},\{5,7\}\})$ :

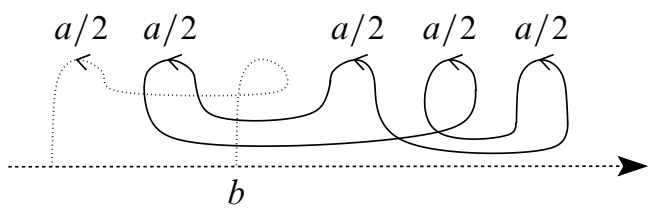

Define now the content of a pair $(w, \wp)$ to be the map $c_{(w, \wp)}: \mathcal{T}_{\mathcal{C}} \rightarrow \mathbb{N}$ which counts the different types of the connected components. For example, the diagram corresponding to the pair

$$
(w, \wp)=(A A A A A B A A,\{\{1,10\},\{2,9\},\{3,5\},\{4,6\},\{7,13\},\{8,12\},\{11,14\}\})
$$

is

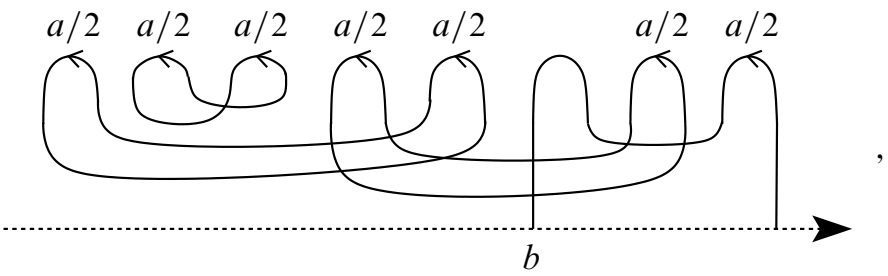

and its corresponding content is thus

$$
c_{(w, \wp)}((\omega, \rho))= \begin{cases}2 & \text { if }(\omega, \rho)=(A A,\{\{1,4\},\{2,3\}\}), \\ 1 & \text { if }(\omega, \rho)=(A A,\{\{1,3\},\{2,4\}\}), \\ 1 & \text { if }(\omega, \rho)=(B A,\{\{1,2\}\}), \\ 0 & \text { otherwise. }\end{cases}
$$

\subsubsection{The "pairings factorize" lemma}

Lemma 5.1.4 Consider some $\tau \in \mathcal{T}$. Then $T_{\tau}=\prod_{\kappa \in \mathcal{T}_{\mathcal{C}}}^{\#}\left(T_{\kappa}\right)^{\# c_{\tau}(\kappa)}$. 
The proof of this lemma will appear shortly, in Section 5.1.6. To illustrate it, consider the case that $\tau=(A A A A A B,\{\{2,9\},\{4,5\},\{8,11\}\})$. In this case, the left hand side of the above equation is equal to

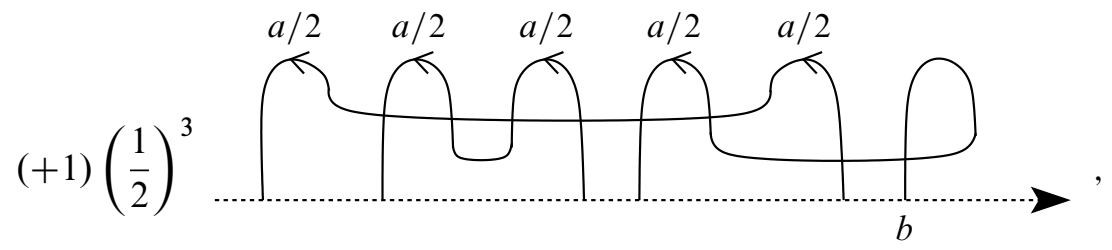

and the right hand side is

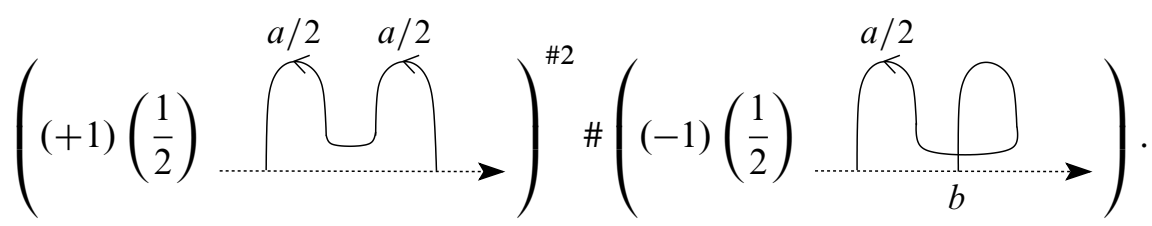

The equality of these two expressions in $\widehat{\mathcal{W}}_{\wedge} \llbracket a, b, \partial_{a}, \partial_{b} \rrbracket$ may be immediately observed.

Applying this lemma to $1+\sum_{\tau \in \mathcal{T}}(1 /|\tau| !) T_{\tau}$, the right-hand-side of Equation (6), we can rewrite the main expression we are computing as

$$
1+\sum_{\substack{\text { content functions } \\
0 \neq c: \mathcal{T}_{\mathcal{C}} \rightarrow \mathbb{N}_{0}}}\left(\begin{array}{c}
\text { number of } \\
\text { pairs } \tau \in \mathcal{T} \\
\text { with content } c
\end{array}\right) \frac{1}{\left(\sum_{\tau \in \mathcal{T}_{\mathcal{C}}} c(\tau)|\tau|\right) !} \prod_{\kappa \in \mathcal{T}_{\mathcal{C}}}^{\#} T_{\kappa}^{\# c(\kappa)} .
$$

The combinatorial factor is computed by the next lemma, whose proof is left as an elementary combinatorial exercise. A detailed proof appeared in the first version of this paper [10].

Lemma 5.1.5 Consider some content function $\tau: \mathcal{T}_{\mathcal{C}} \rightarrow \mathbb{N}_{0}$. The number of pairs $(w, \wp)$ with this content is

$$
\frac{\left(\sum_{\tau \in \mathcal{T}_{\mathcal{C}}} c(\tau)|\tau|\right) !}{\prod_{\kappa \in \mathcal{T}_{\mathcal{C}}}(|\kappa| !)^{c(\kappa)}(c(\kappa)) !}
$$

Substituting this computation into Equation (7), we obtain

$$
1+\sum_{\substack{\text { content functions } \\ 0 \neq c: \mathcal{T}_{\mathcal{C}} \rightarrow \mathbb{N}_{0}}} \prod_{\kappa \in \mathcal{T}_{\mathcal{C}}}^{\#} \frac{1}{c(\kappa) !}\left(\frac{T_{\kappa}}{|\kappa| !}\right)^{\# c(\kappa)} .
$$

This completes the proof of Theorem 5.1.1. 
5.1.6 The proof of Lemma 5.1.4 Our goal is to show that, for $(w, \wp) \in \mathcal{T}$,

$$
T_{(w, \wp)}=\prod_{\kappa \in \mathcal{T}_{\mathcal{C}}}^{\#}\left(T_{\kappa}\right)^{\# c_{(w, \wp)}(\kappa)} .
$$

The right hand side of this equation is just the left hand side, factored into its connected components. The equality is obvious, except for the possibility that the signs may differ. To establish this equality we'll begin by drawing the diagram representing the left-hand side in a canonical way; then we'll push the legs around (using the signed permutation relations) until the diagram is separated into its constituent components. Our task is to keep track of what happens to the sign out the front of the term during this process.

We'll illustrate the discussion with $(w, \wp)=(A A A A A B,\{\{2,9\},\{4,5\},\{8,11\}\})$. We begin with the left-hand side. Construct the corresponding term $T_{(w, \wp)}$ using the graphical approach to $\lambda$ discussed in Section 1.11. In this example,

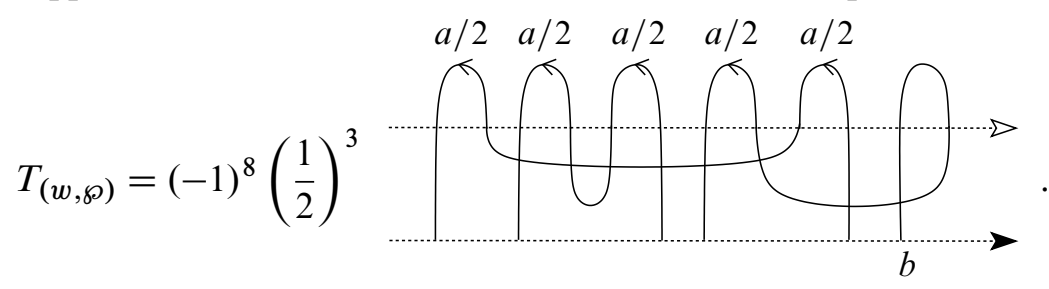

Observe the crucial point:

If we draw the diagram in this fashion, then the sign out the front of the $(\dagger)$ term is precisely a product of a $(-1)$ for every intersection displayed by the drawing.

To connect this with the expression on the right-hand side, we will now separate this diagram into its connected components. To do this we have to perform permutations of the legs. Every time we permute a pair of legs we pick up a $(-1)$, but also pick up an extra intersection point in the drawing. So throughout this process observation $(\dagger)$ still holds. Doing this to our example, we obtain

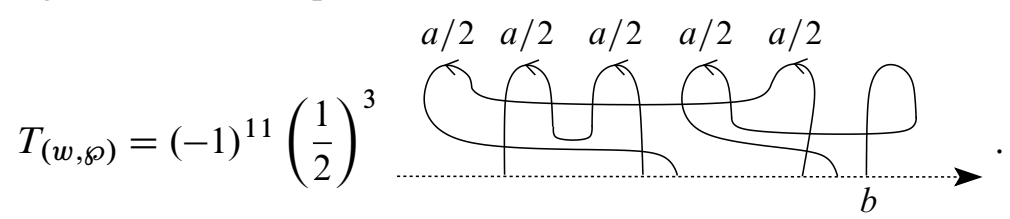

We have now separated the legs of the diagram into disjoint groups corresponding to the different connected components of the diagram.

We finish by fully separating the connected components in the drawing. To be precise, it is clear that we can now do a combination of the following two moves to the drawing 
so as to separate the connected components without affecting the graphical structure of the individual connected components. The moves are

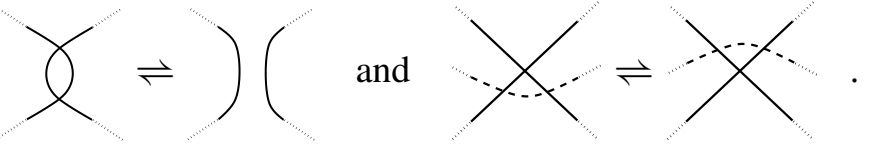

In these diagrams two line styles are used because there are two different connected components involved. Note that these moves only change the number of displayed intersections by an even number, so observation $(\dagger)$ still holds after separating components.

In this case we wind up with

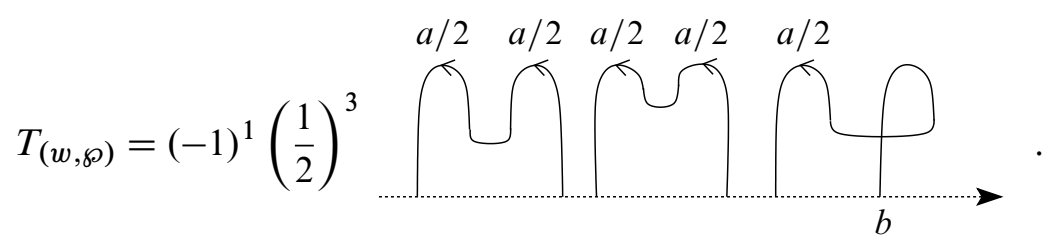

After this factorization procedure the sign that we are left with, then, is a $(-1)$ for every intersection displayed by the drawing. Thus the sign is a $(-1)$ for every self-intersection of the connected components, which exactly gives the right-hand side of Equation (8).

\subsection{The computation of $\sum_{\tau \in \mathcal{T}_{\mathcal{c}}}(1 /|\tau| !) T_{\tau}$}

Our task in this section is to write down the series of all possible terms that can arise by the following procedure:

(1) Putting down a number of copies of the diagrams A and B in some order along a line. If we use $n$ factors in total then we multiply the diagram by $1 / n$ !.

(2) Joining up (with signs) the $\perp$-legs in such a way as to produce a connected diagram (multiplying by a factor of $1 / 2$ for every pair of legs joined up).

We begin by observing that the connected diagrams that can arise in this way fall into exactly four groups:

- The resulting diagram has exactly two remaining legs, and they are both $\perp$-legs. For example,

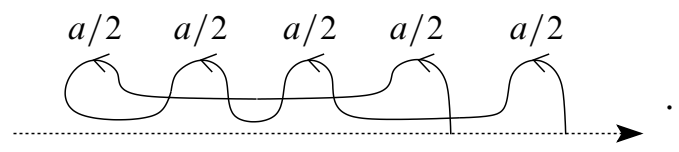


- The resulting diagram has exactly two remaining legs, and they are both $b$-legs.

- The resulting diagram has exactly two remaining legs, one $\perp$-leg and one $b$-leg.

- The resulting diagram has no remaining legs. For example,

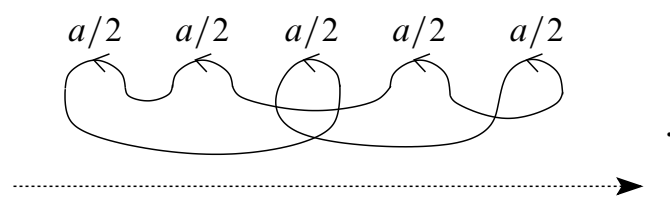

Denote these different contributions in the following way:

$$
\sum_{\tau \in \mathcal{T}_{\mathcal{C}}} \frac{1}{|\tau| !} T_{\tau}=C_{\perp}+C_{\perp b}+C_{b b}+C_{o} .
$$

We'll compute these different contributions in turn, and observe that they correspond to the four series appearing inside the exponential in Theorem 5.0.2.

5.2.1 The contribution $C_{\perp \perp}$ The goal of this subsection is the computation that

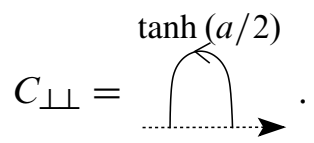

So consider some $n \geq 2$ (the case $n=1$ it is clearer to put in by hand). We wish to compute the contributions from the connected diagrams with 2 legs that we can get by doing signed pairings of the legs of the following term (where the blocks have been numbered for convenience):

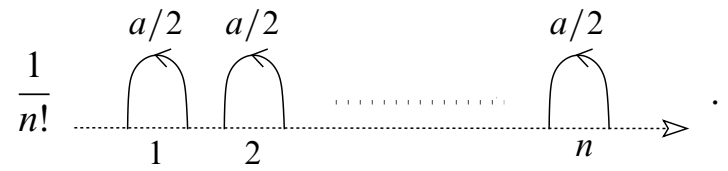

To enumerate the pairings that contribute we'll construct a certain set $\vec{\Gamma}_{n}$. An element of the set $\vec{\Gamma}_{n}$ is a word which uses each of the symbols $\{1,2, \ldots, n\}$ precisely once, where, in addition, each symbol $s$ is decorated by either an arrow pointing to the left $\overleftarrow{s}$ or an arrow pointing to the right $\vec{s}$, for example, $\overrightarrow{2} \overrightarrow{3} \overleftrightarrow{51} \overrightarrow{4} \in \vec{\Gamma}_{5}$. The set $\vec{\Gamma}_{n}$ is defined to be the set of words of this form subject to the single constraint that the first symbol is less than the final symbol. To see that they agree with our definition the reader might like to check that $\left|\vec{\Gamma}_{n}\right|=n ! 2^{n-1}$.

We'll demonstrate how the different pairings correspond with the elements of the sets $\vec{\Gamma}_{n}$ by means of the following example:

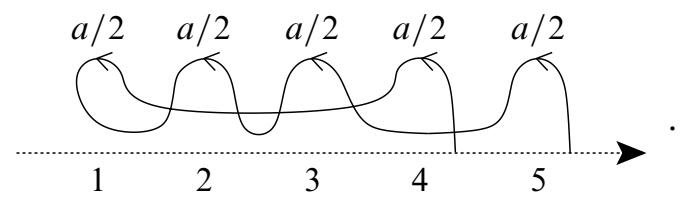


To write down the word corresponding to some pairing, begin at the base of the left-most of the two remaining legs. Now traverse the graph by simply following the edge until you reach the second of the two remaining legs. Write down the symbols $\{1, \ldots, n\}$ in the order in which you visit the different blocks. In this case, you should write down: 41235. Then decorate this word with arrows to record how you traverse each block (whether from left to right, or from right to left). (Take care not to confuse this arrow with the arrow which locally orients the edges around the $a$ labels.) The decoration in this example is $\overleftarrow{4} \overleftarrow{1} \overleftrightarrow{2} \overrightarrow{3} \overrightarrow{5}$

This word contains sufficient instructions for uniquely reconstructing the pairing, so we get precisely one of the relevant pairings for each element of $\vec{\Gamma}_{n}$.

Given some word $w \in \vec{\Gamma}_{n}$, let $\gamma_{w}$ denote the corresponding contribution to $C_{\perp \perp}$ (that is, including the factorial out the front as well as the signs that arise from the gluing and a factor of $(1 / 2)$ for every joined pair). For example,

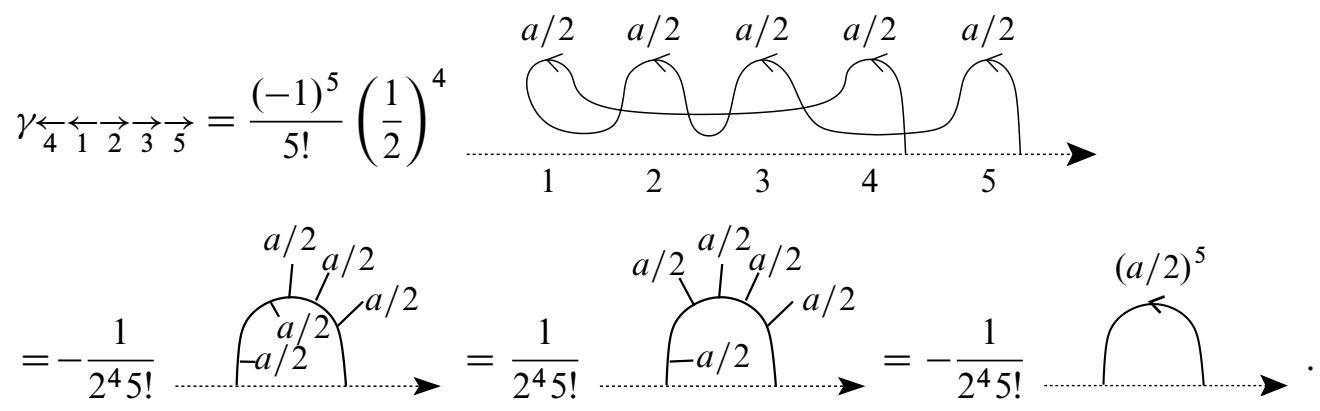

With this definition we can organize the contributions that we are seeking to compute in the following way:

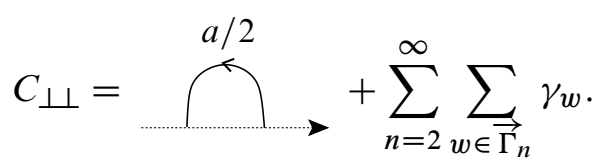

Now observe that, for some fixed $n$, all the $\gamma_{w}$, for $w \in \vec{\Gamma}_{n}$, are equal, up to sign:

$$
\gamma_{w}= \pm \frac{1}{2^{n-1} n !} \overbrace{\longrightarrow}^{(a / 2)^{n}} .
$$

The only complication, then, in computing the piece $\left(\sum_{w \in \vec{\Gamma}_{n}} \gamma_{w}\right)$ in Equation (10), is to determine the signs of the various $\gamma_{w}$. Those signs are determined by the next lemma.

To state the lemma precisely, we need some notation. Let $\Gamma_{n}$ denote the set of words in the symbols $\{1, \ldots, n\}$ with the property that the right-most symbol in a word has 
greater value than the left-most symbol. There is an obvious $2^{n}$-to- 1 forgetful map $f_{\Gamma}: \vec{\Gamma}_{n} \rightarrow \Gamma_{n}$. Define the descent of a word $w \in \Gamma_{n}$, denoted $d(w)$, to be the number of times the value of the symbol decreases as you scan the word from left to right.

Lemma 5.2.2 Let $n \geq 2$ and let $w \in \vec{\Gamma}_{n}$. Then

$$
\gamma_{w}=(-1)^{d\left(f_{\Gamma}(w)\right)} \frac{1}{2^{n-1} n !} \bigcap_{\longrightarrow}^{(a / 2)^{n}} .
$$

The proof of this lemma is at the end of this subsection. Substituting the computation into Equation (10), we find that

$$
\begin{aligned}
C_{\perp \perp} & =\bigcap_{\longrightarrow}^{a / 2}+\sum_{n=2}^{\infty} \sum_{w \in \Gamma_{n}}((-1)^{d\left(f_{\Gamma}(w)\right)} \frac{1}{2^{n-1} n !} \overbrace{\longrightarrow}^{(a / 2)^{n}}) \\
& =\bigcap^{\infty / 2}\left(\frac{2 \sum_{w \in \Gamma_{n}}(-1)^{d(w)}}{n !}\right)
\end{aligned}
$$

Note that when $n$ is even the corresponding term is zero (because of AS relations). So the result is

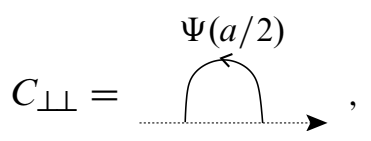

where $\Psi(x)$ is the formal power series defined by $\Psi(x)=\sum_{n=1}^{\infty}(\psi(n) / n !) x^{n}$, with

$$
\psi(n)= \begin{cases}1 & \text { if } n=1 \\ 2 \sum_{w \in \Gamma_{n}}(-1)^{d(w)} & \text { if } n>1 \text { and } n \text { is odd } \\ 0 & \text { if } n>1 \text { and } n \text { is even }\end{cases}
$$

The required computation, Equation (9), is completed by the following proposition.

Proposition 5.2.3 $\Psi(x)=\tanh (x)$.

Proof We'll begin by replacing $\psi(n)$ with a function that is easier to use. For every $n \geq 1$ let $\Sigma_{n}$ denote the set of words that can be made using each of the symbols $\{1, \ldots, n\}$ exactly once (with no restrictions on order) and define

$$
\phi(n)=\sum_{w \in \Sigma_{n}}(-1)^{d(w)} .
$$


Let $v: \Sigma_{n} \rightarrow \Sigma_{n}$ be the involution of $\Sigma_{n}$ which writes a word in its reverse order. Define the descent $d(w)$ of a word in the obvious way. Notice that

$$
d(v(w))= \begin{cases}+d(w) & \text { if } n \text { is odd } \\ -d(w) & \text { if } n \text { is even }\end{cases}
$$

Thus

$$
\phi(n)= \begin{cases}1 & \text { if } n=1, \\ 2 \sum_{w \in \Gamma_{n}}(-1)^{d(w)} & \text { if } n>1 \text { and } n \text { is odd, } \\ 0 & \text { if } n>1 \text { and } n \text { is even. }\end{cases}
$$

In other words, $\phi(n)=\psi(n)$, and our task is to calculate the power series

$$
\Psi(x)=\sum_{n=1}^{\infty} \frac{\phi(n)}{n !} x^{n} .
$$

We'll calculate this power series by writing down a recursion relation which determines the function $\phi(n) / n$ !, and then we'll identify a power series whose coefficients solve the recursion relation.

To deduce the appropriate recursion relation we'll partition the set $\Sigma_{n}$ according to the position of the symbol $n$. Let $\Sigma_{n}^{i} \subset \Sigma_{n}$ denote the subset consisting of the words where the symbol $n$ appears in position $i$. Then, for $n \geq 3$ :

$$
\begin{aligned}
\frac{\phi(n)}{n !} & =\frac{1}{n !} \sum_{i=1}^{n}\left(\sum_{w \in \Sigma_{n}^{i}}(-1)^{d(w)}\right) \\
& =-\frac{1}{n !} \sum_{i=2}^{n-1}\left(\begin{array}{c}
n-1 \\
i-1
\end{array}\right) \phi(i-1) \phi(n-i) \\
& =-\frac{1}{n} \sum_{i=2}^{n-1} \frac{\phi(i-1)}{(i-1) !} \frac{\phi(n-i)}{(n-i) !} .
\end{aligned}
$$

Now observe that this recursion relation, together with the initial conditions $\phi(1)=1$ and $\phi(2)=0$, completely determines the sequence $\phi(n)$. It follows from this recursion relation that $\Psi(x)$ is the unique power series satisfying the equation

$$
\frac{\mathrm{d}}{\mathrm{d} x}[\Psi(x)]=1-\Psi(x)^{2},
$$

with initial terms $\Psi(x)=x+$ (terms of degree at least 3 ). Thus, $\Psi(x)=\tanh (x)$.

Proof of Lemma 5.2.2 Consider the diagram arising from the pairing corresponding to some word $w \in \vec{\Gamma}_{n}$. If we draw the pairing canonically (as described in Section 
1.11) then the sign of the resulting term is precisely $(-1)$ raised to the number of intersections displayed in the drawing of the diagram. For example,

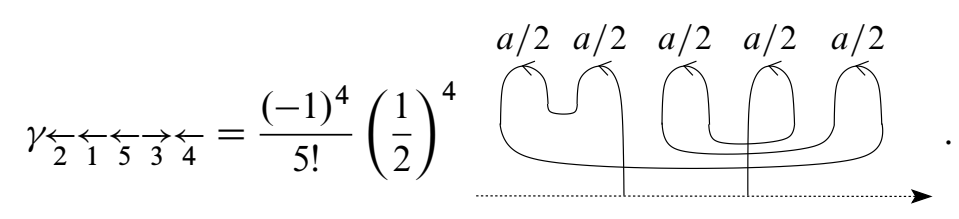

We'll put such a drawing into a standard form with two steps. The first step is to add permutations to the top of the drawing so that the tops of the blocks appear in the same order as they appear in the word $w$. Notice that a single such permutation introduces 4 intersections into the diagram, so it is still true, after such a move, that the sign of the term is precisely $(-1)$ raised to the number of intersections displayed in the drawing of the diagram. For example,

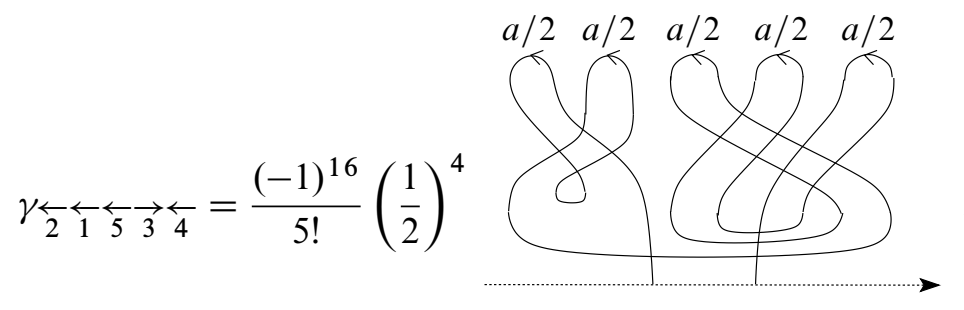

Now the second step. Notice that as you traverse the edge from the base of the left leg to the base of the right leg, then some factors are traversed from left to right (the 4-th factor from the left, above), while some factors are traversed from right to left (the other factors). The second and final step in the procedure to put the diagram into standard form is to add twists to the maxima in order that every factor is traversed from left to right. Because this move simultaneously introduces an extra intersection, and an extra factor of (-1) (from a use of an AS relation), it is still true that the sign of the term is just $(-1)$ raised to the number of intersections displayed in the drawing. If we apply this procedure to our example we obtain

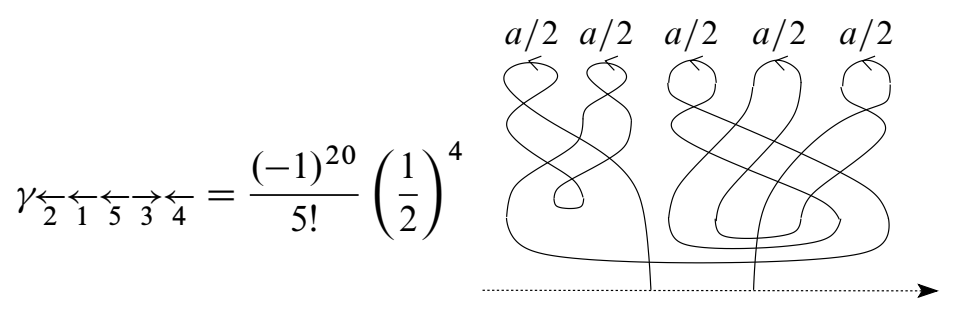

Notice that after these two steps, the initial diagram has been transformed into the following standard form: 


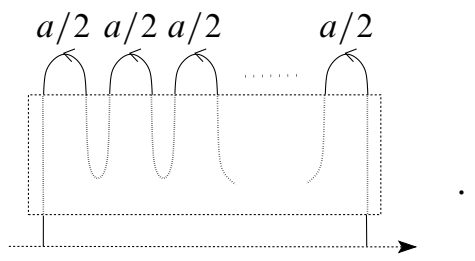

The sign of the corresponding term, which is what we are calculating, is precisely $(-1)$ raised to the number of intersection points displayed within the box in the drawing that we have just obtained.

To count the intersections: Notice that the dashed box cuts the edge up into pieces. We'll call the pieces inside the box the arcs. The arcs correspond directly to the arcs that were introduced when this diagram was created in the operation $\lambda$.

The number of intersections of two different arcs must be even (because of how their boundary points are arranged around the outside of the box). It remains, then, to count the number of self-intersection points of these arcs. Well, the arcs had no self-intersections when they were added in by $\lambda$, so we just have to trace how many self-intersection points were created by our procedure. Self-intersections of the arcs can only be created in the first step of our procedure, when a permutation is added to the top of the diagram. There will be exactly one of these for every case of two factors consecutive in $w$, with the factor appearing later in $w$ having the smaller value. Thus the sign is $(-1)^{d(w)}$.

5.2.4 The contribution $C_{o}$ The goal of this subsection is the computation that

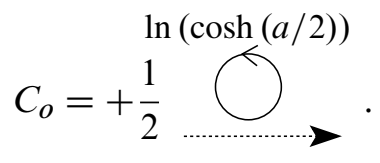

So consider some $n \geq 2$ (the $n=1$ case is easily observed to be zero). In this subsection we wish to compute the contributions from the connected diagrams with zero legs that we can get by doing signed pairings of the legs of the term

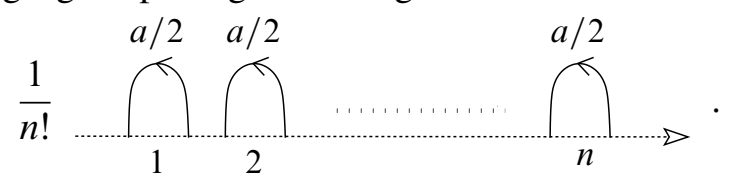

In this case, to enumerate the contributions we'll employ a set $\vec{\Xi}_{n}$. This set consists of the words that can be made by using each of the symbols $\{1, \ldots, n\}$ precisely once, such that the left-most symbol is a 1, and where every symbol $s$ except the initial 1 is decorated by either an arrow pointing to the right $\vec{s}$ or an arrow pointing to the left $\overleftarrow{s}$. For example, $1 \overrightarrow{3} \overrightarrow{5} \overrightarrow{4} \overleftarrow{2} \in \vec{\Xi}_{5}$. Let $\Xi_{n}$ denote the set defined in the same 
way but without the arrow decorations and let $f_{\Xi}: \vec{\Xi}_{n} \rightarrow \Xi_{n}$ denote the corresponding $2^{n-1}-$ to-1 forgetful map.

Consider, then, some pairing which uses all the available legs and results in exactly one connected component. For example,

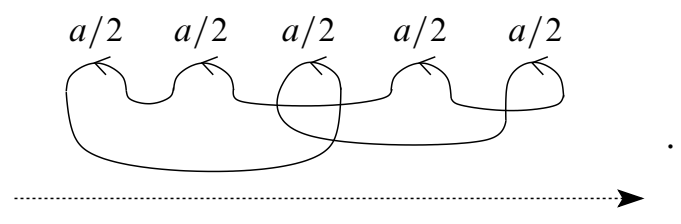

The word that corresponds to such a gluing is determined in the following way. To begin, ignore the arc that terminates on the left-hand side of the left-most block:

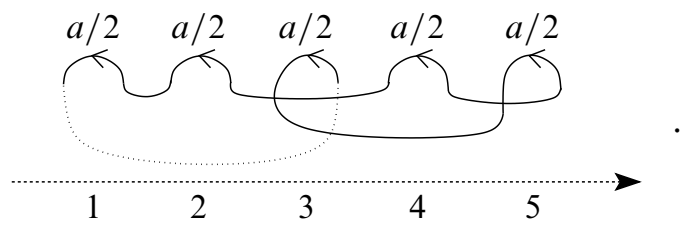

Now traverse the graph, starting with the left-most block, writing down the order in which blocks are visited together with the corresponding directions. The example given leads to $1 \overrightarrow{2} \overrightarrow{4} \overleftrightarrow{5} \overrightarrow{3}$. This word contains sufficient information to reconstruct the pairing, so we have just set up a bijection between the set $\vec{\Xi}_{n}$ and the set of pairings that contribute to this case.

Given some word $w \in \vec{\Xi}_{n}$ let $\xi_{w}$ denote the corresponding contribution.

Lemma 5.2.5 Let $n \geq 2$ and let $w \in \vec{\Xi}_{n}$. Then

$$
\xi_{w}=(-1)^{d\left(f_{\Xi}(w)\right)} \frac{1}{2^{n} n !} \bigcirc_{>}^{(a / 2)^{n}} .
$$

This is proved in exactly the same way as the proof of Lemma 5.2.2. The one substantial difference is that this term has an extra factor of 2 in the denominator (in comparison with Lemma 5.2.2) because this term has an extra arc attached. (So $\lambda$ introduces an extra factor of $1 / 2$.) 
Now we can compute the contribution $C_{o}$. To begin, note that the term that arises in the $n=1$ case is zero. We calculate

$$
\begin{aligned}
C_{o}=\sum_{n=2}^{\infty}\left(\frac{2^{n-1} \sum_{w \in \Xi_{n}}(-1)^{d(w)}}{2^{n} n !}\right) & =\frac{1}{2} \sum_{n=2}^{\infty}\left(\frac{1}{n} \frac{\phi(n-1)}{(n-1) !}\right)
\end{aligned}
$$

where $\left(\int \Psi\right)(x)$ denotes

$$
\sum_{n=2}^{\infty} \frac{1}{n} \frac{\phi(n-1)}{(n-1) !} x^{n} .
$$

This is the unique power series with zero constant term whose formal derivative is $\Psi(x)=\tanh x$. That power series is $\left(\int \Psi\right)(x)=\ln \cosh x$, as claimed.

5.2.6 The contribution $C_{b b}$ We now wish to compute the contribution of pairings which lead to connected diagrams with exactly two $b$-legs. For example,

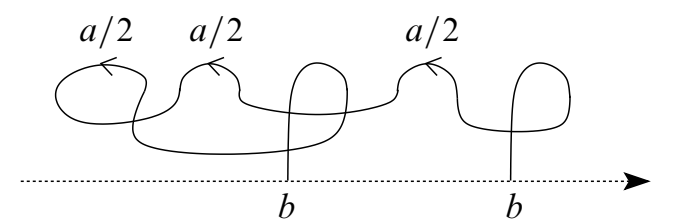

The set which enumerates these gluings is the following $\vec{\Omega}_{n}$. The elements of $\vec{\Omega}_{n}$ are certain words that use each of the symbols $\{1, \ldots, n\}$ precisely once and where each symbol except the first and last symbols in the word is decorated by an arrow. The set $\vec{\Omega}_{n}$ is defined to be all words of this type with the property that the last symbol is greater than the first symbol.

To write down the word corresponding to some gluing, traverse the graph, starting at the left-most of the 2 legs, writing down the order that blocks are encountered as you traverse (decorating with the appropriate arrow). For example, the gluing above corresponds with the word $3 \overleftrightarrow{12} \overrightarrow{4} 5 \in \vec{\Omega}_{5}$. Let $\Omega_{n}$ be defined in exactly the same way as $\vec{\Omega}_{n}$, but without using the arrow decorations. Let $f_{\Omega}: \vec{\Omega}_{n} \rightarrow \Omega_{n}$ denote the $2^{n-2}$-to-1 forgetful map. Let $\omega_{w}$ denote the contribution corresponding to some word $w \in \vec{\Omega}_{n}$. We leave the proof of the following lemma as an exercise for the reader. 
Lemma 5.2.7 Let $n \geq 2$ and let $w \in \vec{\Omega}_{n}$. Then

$$
\omega_{w}=-(-1)^{d\left(f_{\Omega}(w)\right)} \frac{1}{2^{n-1} n !} \bigcap_{b}^{(a / 2)^{n-2}} .
$$

With this lemma we can now compute, as required, that

$$
\begin{aligned}
C_{b b}=-\sum_{n=2}^{\infty}\left(\frac{2^{n-2}}{2^{n-1} n !} \sum_{w \in \Gamma_{n}}(-1)^{d(w)}\right) \overbrace{b}^{(a / 2)^{n-2}} \\
=-\left(\frac{1}{2}\right)^{2} \sum_{n=2}^{\infty}\left(\frac{\psi(n)}{n !}\right) \overbrace{b}^{(a / 2)^{n-2}}=-\left(\frac{1}{2}\right)^{2} \overbrace{b}^{\frac{(a / 2)^{2}}{b}} .
\end{aligned}
$$

5.2.8 The contribution $C_{\mid b}$ To enumerate these contributions the set we'll use will be $\vec{\Delta}_{n}$, which we define to be the set of words which use each one of the symbols $\{1, \ldots, n\}$ exactly once, and where every symbol except the last symbol is decorated by an arrow. For example, $\overleftarrow{4} \overrightarrow{2} \overleftarrow{3} 1 \in \vec{\Delta}_{5}$.

To write down the word that corresponds with a given pairing, traverse the diagram, starting at the $\perp$-leg and proceeding until you reach the $b$-leg As you traverse, record the order in which you visit the different blocks and the directions in which you travel as you visit the blocks. For example, the pairing

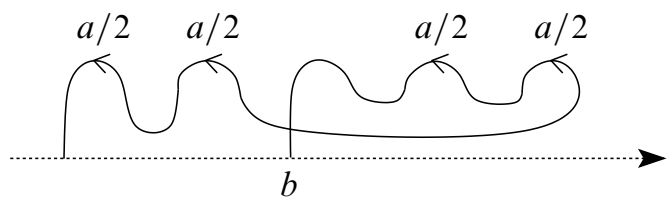

corresponds with the word $\overrightarrow{1} \overrightarrow{2} \overleftarrow{5} \overleftarrow{4} 3 \in \vec{\Delta}_{5}$. For some $w \in \vec{\Delta}_{n}$, let $\delta_{w}$ denote the corresponding term. Let $f_{\Delta}: \vec{\Delta}_{n} \rightarrow \Sigma_{n}$ denote the $2^{n-1}$-to-1 forgetful map.

Lemma 5.2.9 Let $n \geq 2$ and let $w \in \vec{\Delta}_{n}$. Then

$$
\delta_{w}=-(-1)^{d\left(f_{\Delta}(w)\right)} \frac{1}{2^{n-1} n !} \overbrace{b}^{(a / 2)^{n-1}} .
$$


We'll leave this lemma as an exercise. With it we can calculate the claimed result:

$$
\begin{aligned}
C_{\mid b}=-\sum_{n=1}^{\infty} \sum_{w \in \vec{\Delta}_{n}}(-1)^{d(\delta(w))} \frac{1}{2^{n-1} n !} \overbrace{b}^{(a / 2)^{n-1}} \\
=-\sum_{n=1}^{\infty} \frac{\sum_{w \in \Sigma_{n}}(-1)^{d(w)}}{n !} \overbrace{b}^{(a / 2)^{n-1}}=-\overbrace{b / 2)^{-1} \Psi(a / 2)}^{(a)} .
\end{aligned}
$$

\section{Computing the operator product II}

In this section we'll complete the computation of the series $[\mathcal{X}]_{b, \partial_{b}=0}$. The final result we'll obtain was stated earlier as Theorem 4.1.2.

\subsection{Proof of Theorem 4.1.2}

A direct substitution of the previous section's result (Theorem 5.0.2) into $\mathcal{X}$ (as defined in Theorem 4.1.1) tells us that $[\mathcal{X}]_{b, \partial_{b}=0}$ is equal to

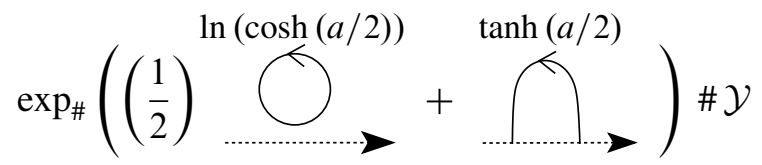

where $\mathcal{Y}$ is equal to

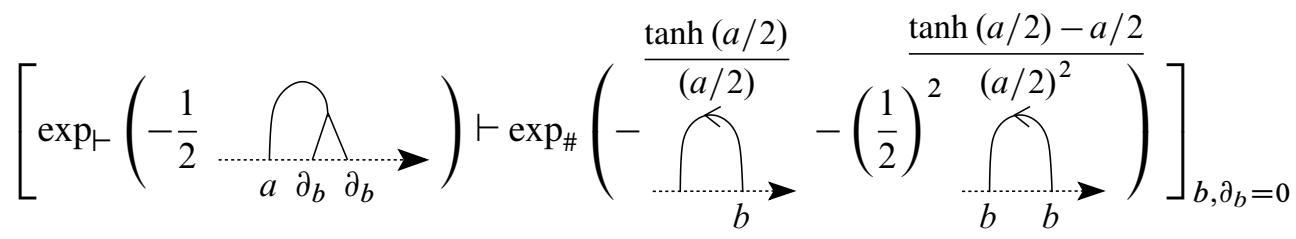

This series $\mathcal{Y}$ will be computed with the following lemma, whose proof occupies the remainder of this section (following the proof we are in right now). Observe that the minus that appears inside the first term has been removed by appropriately choosing the orientation on the corresponding edge.

Lemma 6.1.1 Let $Y(a)$ be a power series containing only even powers of $a$ and let $Z(a)$ be a power series containing only odd powers of $a$. Then 


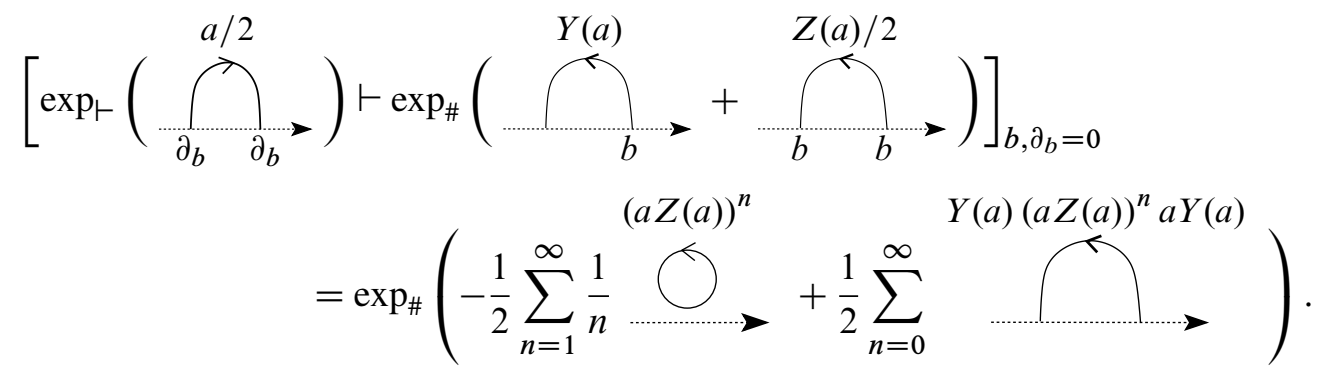

We'll apply this lemma directly to the expression for $\mathcal{Y}$ given above. To specialize to this case we set

$$
\begin{aligned}
& Y(a)=-\frac{\tanh (a / 2)}{a / 2}, \\
& Z(a)=-\frac{1}{2} \frac{\tanh (a / 2)-(a / 2)}{(a / 2)^{2}} .
\end{aligned}
$$

The expressions appearing in the right-hand-side of the lemma become

$$
\sum_{n=1}^{\infty} \frac{1}{n}(Z(a) a)^{n}=\sum_{n=1}^{\infty} \frac{1}{n}\left(1-\frac{\tanh (a / 2)}{a / 2}\right)^{n}=-\ln \left(\frac{\tanh (a / 2)}{a / 2}\right),
$$

$$
\text { and } \begin{aligned}
\frac{1}{2} \sum_{n=0}^{\infty} Y(a)(a Z(a))^{n} a Y(a) & =\sum_{n=0}^{\infty} \frac{\tanh (a / 2)}{a / 2}\left(1-\frac{\tanh (a / 2)}{a / 2}\right)^{n} \frac{a}{2} \frac{\tanh (a / 2)}{a / 2} \\
& =\tanh (a / 2) .
\end{aligned}
$$

Thus the conclusion of the lemma is that $\mathcal{Y}$ is equal to

$$
\exp _{\#}(\left(\frac{1}{2}\right) \bigcirc_{>}^{\ln \left(\frac{\tanh (a / 2)}{(a / 2)}\right)}-\overbrace{-}^{\tanh (a / 2)}
$$

Substituting this computation into (12) completes the proof of Theorem 4.1.2.

\subsection{Proof of Lemma 6.1.1}

Our final task is the combinatorial computation of the following expression:

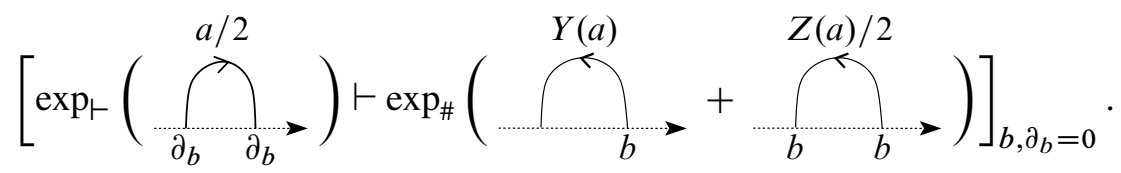


To begin, note that, because we set $b$ and $\partial_{b}$ to zero at the end of the calculation, there will only be contributions from those terms arising from the expansion with the property that the number of $\partial_{b}$ legs in the first factor is equal to the number of $b$ legs in the second factor. For example, a typical contributing term is
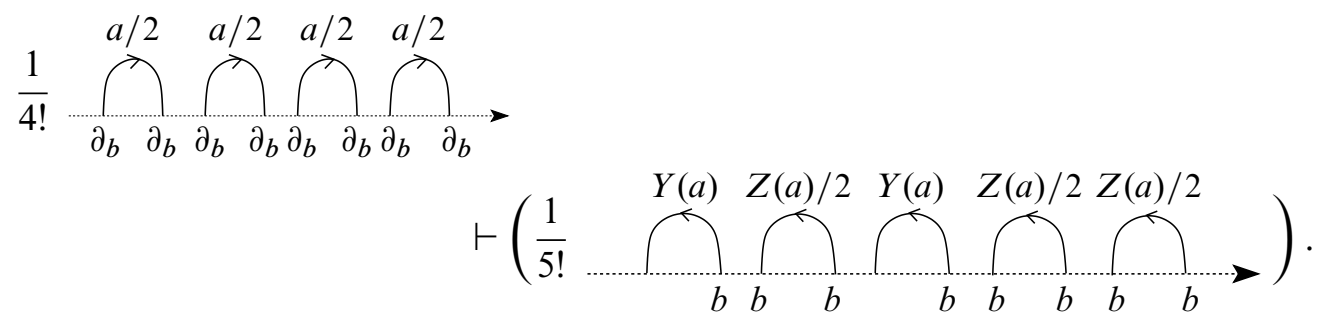

We'll briefly recall how to compute such a product using the graphical method described in Section 3.3. We begin by drawing a grid over an orienting line, placing the legs of the first factor in order up the left-hand side of the grid, and the legs of the second factor in order along the top of the grid. Because we are going to set $b$ and $\partial_{b}$ to zero at the end of the calculation, we will get exactly one contribution for every different way of wiring all the $\partial_{b}$-legs to all the $b$-legs. In other words, we get exactly one contribution for every bijection $\phi:\{1, \ldots, 8\} \rightarrow\{1, \ldots, 8\}$.

Consider the example of the bijection

$$
\left(\begin{array}{llllllll}
1 & 2 & 3 & 4 & 5 & 6 & 7 & 8 \\
1 & 5 & 2 & 7 & 4 & 6 & 8 & 3
\end{array}\right)
$$

To construct the corresponding contribution we, first of all, wire up the grid using the bijection, and then multiply the resulting diagram by $(1 / 4 !)(1 / 5 !)(-1)^{x}$, where $x$ denotes the number of intersections displayed within the grid. In this case $x=32$. The exact contribution is shown in Figure 13.

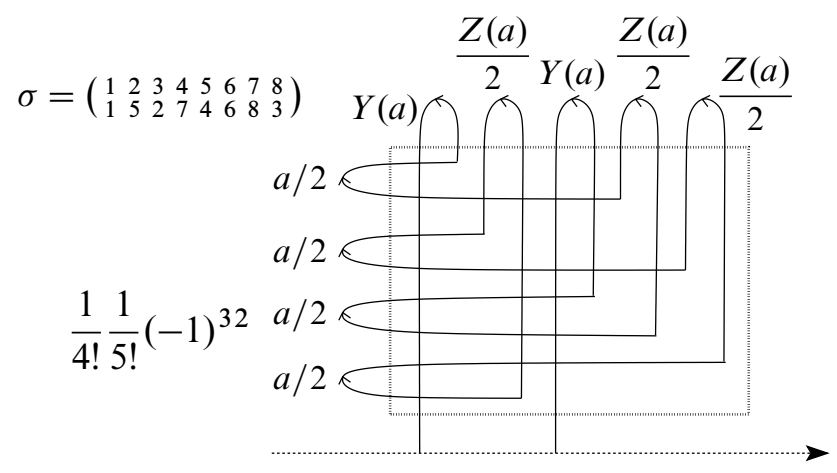

Figure 13: The contribution corresponding to the bijection $\sigma$ 
6.2.1 The series is an exponential of connected terms We'll begin by defining a set $\nabla$ which will index the different terms that arise when Expression (13) is evaluated. The elements of $\nabla$ are triples $(n, w, \sigma)$, where $n$ is a positive integer, where $w$ is a word in the symbols $Y$ and $Z$ such that $(\# Y)+2(\# Z)=2 n$, and where $\sigma$ is a bijection $\sigma:\{1,2, \ldots, 2 n\} \rightarrow\{1,2, \ldots, 2 n\}$. Given some triple $(n, w, \sigma) \in \nabla$, let $T_{(n, w, \sigma)}$ denote the corresponding term. For example, the term we constructed in Figure 13 is precisely

$$
T_{\left(4, Y Z Y Z Z,\left(\begin{array}{llllllll}
1 & 2 & 3 & 4 & 5 & 6 & 7 & 8 \\
1 & 5 & 2 & 7 & 4 & 6 & 8 & 3
\end{array}\right)\right)} \cdot
$$

Let $\nabla_{\mathcal{C}} \subset \nabla$ denote the subset of elements of $\nabla$ whose corresponding diagram is connected. (For example, the case just treated is not an element of this subset.) We'll omit the proof of the following proposition, which is a tedious combinatorial argument closely analogous to the proof of Theorem 5.1.1.

Proposition 6.2.2 Expression (13) is equal to $\exp _{\#}\left(\sum_{\tau \in \nabla_{\mathcal{C}}} T_{\tau}\right)$.

6.2.3 The computation of $\sum_{\tau \in \nabla_{\mathcal{C}}} T_{\tau}$ We can group this sum into two contributions $\sum_{\tau \in \nabla_{\mathcal{C}}} T_{\tau}=C_{0}+C_{2}$, where:

- $C_{0}$ denotes the series of terms $T_{\tau}$ whose underlying diagrams are connected and have no legs.

- $C_{2}$ denotes the series of terms $T_{\tau}$ whose underlying diagrams are connected and have exactly 2 legs.

These classes are the only combinatorial possibilities. In the next section $C_{0}$ will be calculated to be

$$
C_{0}=-\frac{1}{2} \sum_{n=1}^{\infty} \frac{1}{n} \bigcirc_{-}^{(a Z(a))^{n}} .
$$

This fact, together with the fact that

$$
C_{2}=\frac{1}{2} \sum_{n=0}^{\infty} \overbrace{\longrightarrow}^{Y(a)(a Z(a))^{n} a Y(a)},
$$

completes the proof of the Lemma 6.1.1. The computation of $C_{2}$ is closely analogous to the computation of $C_{0}$, and will be omitted here. A detailed discussion appeared in the first version of this paper [10]. 
6.2.4 The contribution $C_{0}$ We'll begin by defining a set $\vec{\Phi}_{n}$ which will index the terms (with $\# Z=n$ ) which contribute to $C_{0}$. The elements of $\vec{\Phi}_{n}$ will be pairs $\left(w_{1}, w_{2}\right)$ of words, the first word $w_{1}$ using each of the symbols $\{1, \ldots, n\}$ exactly once, and the second word $w_{2}$ using each of the symbols $\{2, \ldots, n\}$ exactly once. In addition, every symbol $s$ of the first word $w_{1}$ is decorated by either an arrow pointing to the left $\overleftarrow{s}$ or an arrow pointing to the right $\vec{s}$, and every symbol $s$ of the second word $w_{2}$ is decorated by either an arrow pointing up $s \uparrow$ or an arrow pointing down $s \downarrow$. The set $\vec{\Phi}_{n}$ is defined to be the set of all such pairs. For example, $(\overrightarrow{4} \overleftarrow{1} \stackrel{\leftarrow}{32}, 2 \uparrow 3 \downarrow 4 \downarrow) \in \vec{\Phi}_{4}$.

We'll now explain how to write down the pair of words corresponding to some contribution to $C_{0}$. The explanation will be illustrated by the example shown in Figure 14 . Start at the top-most factor $a / 2$. The exact point referred to is decorated in the figure with a bullet.

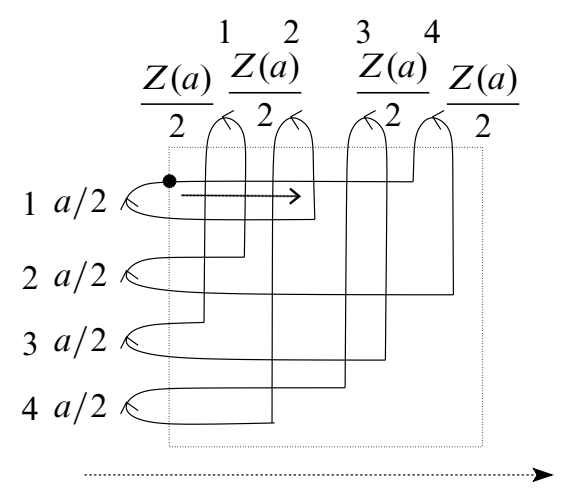

Figure 14: How a gluing determines a pair of words

Now traverse the diagram until you return to where you started (the arrow in the example indicates how to begin this traverse). The first word records the order and direction in which you encounter the factors written along the top line as you traverse (in this example the corresponding word is $\overrightarrow{4} \stackrel{\leftarrow}{4} \stackrel{\leftarrow}{\longleftrightarrow}$ ), and the second word records the order and direction in which you encounter the factors written down the left-hand side (in this case, $2 \uparrow 3 \downarrow 4 \downarrow)$.

Given some element $\left(w_{1}, w_{2}\right) \in \vec{\Phi}_{n}$, let $\phi_{\left(w_{1}, w_{2}\right)}$ denote the corresponding contribution (including the appropriate factorials and signs) to $C_{0}$. The series to be calculated can now be written

$$
C_{0}=\sum_{n=1}^{\infty} \sum_{\left(w_{1}, w_{2}\right) \in \vec{\Phi}_{n}} \phi_{\left(w_{1}, w_{2}\right)}
$$


This calculation is greatly simplified by the observation that, for a fixed $n$, all the $\phi_{\left(w_{1}, w_{2}\right)}$, for $\left(w_{1}, w_{2}\right) \in \vec{\Phi}_{n}$, are precisely equal. This fact is part of the following lemma, whose proof appears later in this section.

Lemma 6.2.5 Consider some $n \geq 1$ and some $\left(w_{1}, w_{2}\right) \in \vec{\Phi}_{n}$. Then

$$
\phi_{\left(w_{1}, w_{2}\right)}=-\frac{1}{n ! n !} \bigcirc_{\longrightarrow}^{((a / 2)(Z(a) / 2))^{n}}
$$

With this information in hand, $C_{0}$ is easily computed:

$$
C_{0}=(-1) \sum_{n=1}^{\infty}\left|\vec{\Phi}_{n}\right| \frac{1}{n ! n !} \bigcirc_{-}^{((a / 2)(Z(a) / 2))^{n}}=-\frac{1}{2} \sum_{n=1}^{\infty} \frac{1}{n} \bigcirc_{-}^{(a Z(a))^{n}}
$$

This is the computation we claimed at the start of this section.

Proof of Lemma 6.2.5 We'll begin by introducing some notation. Given a gluing datum $\left(w_{1}, w_{2}\right) \in \vec{\Phi}_{n}$, let $D_{\left(w_{1}, w_{2}\right)}$ denote the series of diagrams represented by the drawing you get when you wire up a grid according to $\left(w_{1}, w_{2}\right)$. Let $x\left(w_{1}, w_{2}\right)$ denote the number of intersections displayed by that drawing. According to these definitions, the corresponding contribution is written

$$
\phi_{\left(w_{1}, w_{2}\right)}=\frac{1}{n ! n !}(-1)^{x\left(w_{1}, w_{2}\right)} D_{\left(w_{1}, w_{2}\right)} .
$$

This proof is based on two moves, which we'll call R-moves (for tRansposition) and $\mathrm{W}$-moves (for tWist), that we can perform on gluing data:

$$
\left(w_{1}, w_{2}\right) \stackrel{\text { R-move }}{\longrightarrow}\left(w_{1}^{\prime}, w_{2}^{\prime}\right) \text { and }\left(w_{1}, w_{2}\right) \stackrel{\text { W-move }}{\longrightarrow}\left(w_{1}^{\prime}, w_{2}^{\prime}\right),
$$

whose key property is that they have no effect on the corresponding term:

$$
\frac{1}{n ! n !}(-1)^{x\left(w_{1}, w_{2}\right)} D_{\left(w_{1}, w_{2}\right)}=\frac{1}{n ! n !}(-1)^{x\left(w_{1}^{\prime}, w_{2}^{\prime}\right)} D_{\left(w_{1}^{\prime}, w_{2}^{\prime}\right)} .
$$

We'll begin by introducing these two moves and establishing that the key property holds for them. 
R-moves This move is transposition of adjacent columns or adjacent rows. Here is an example of an R-move, (where the arcs which are unaltered by the move have not been drawn in):
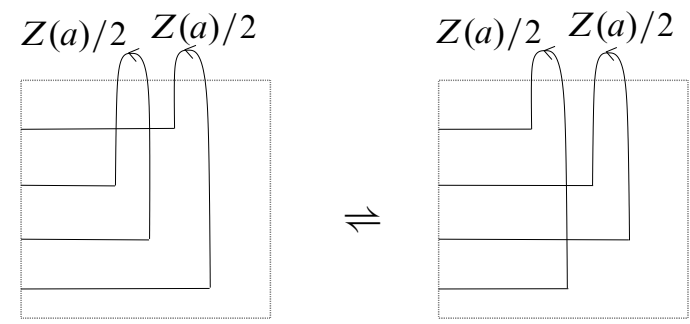

This move can only change the number of displayed intersections by an even number (indeed, observe that the relative positions of the ends of all arcs down the left-hand edge of the grid is unaltered by this move). Thus

$$
\begin{aligned}
& \frac{1}{n ! n !}(-1)^{x\left(w_{1}, w_{2}\right)} D_{\left(w_{1}, w_{2}\right)} \\
= & \frac{1}{n ! n !}(-1)^{x\left(w_{1}, w_{2}\right)} D_{\left(w_{1}^{\prime}, w_{2}^{\prime}\right)} \quad\left(\text { as } D_{\left(w_{1}^{\prime}, w_{2}^{\prime}\right)}=D_{\left(w_{1}, w_{2}\right)}\right) \\
= & \frac{1}{n ! n !}(-1)^{x\left(w_{1}^{\prime}, w_{2}^{\prime}\right)} D_{\left(w_{1}^{\prime}, w_{2}^{\prime}\right)} \quad\left(\text { as } x\left(w_{1}^{\prime}, w_{2}^{\prime}\right)=x\left(w_{1}, w_{2}\right)+\text { an even number }\right) .
\end{aligned}
$$

W-moves This move is a "half-twist" of a single column or row:
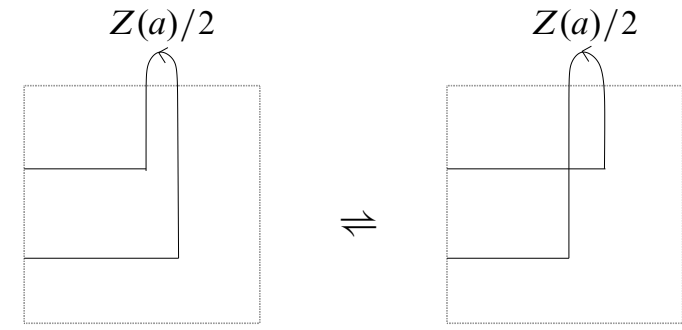

Note in this case that $D_{\left(w_{1}^{\prime}, w_{2}^{\prime}\right)}=(-1) D_{\left(w_{1}, w_{2}\right)}$, (using the fact that $Z(a)$ is assumed to only have odd powers of $a$ ), so

$$
\begin{aligned}
& \frac{1}{n ! n !}(-1)^{x\left(w_{1}, w_{2}\right)} D_{\left(w_{1}, w_{2}\right)} \\
= & \frac{1}{n ! n !}(-1)^{x\left(w_{1}, w_{2}\right)}(-1) D_{\left(w_{1}^{\prime}, w_{2}^{\prime}\right)} \\
= & \frac{1}{n ! n !}(-1)^{x\left(w_{1}^{\prime}, w_{2}^{\prime}\right)} D_{\left(w_{1}^{\prime}, w_{2}^{\prime}\right)} \quad\left(\text { as } D_{\left(w_{1}^{\prime}, w_{2}^{\prime}\right)}=(-1) D_{\left(w_{1}, w_{2}\right)}\right) \\
& \left.\left(w_{1}^{\prime}, w_{2}^{\prime}\right)=x\left(w_{1}, w_{2}\right) \pm 1\right),
\end{aligned}
$$

as required. 
With these moves in hand, we can turn to the general argument. The simple idea is to show that we can transform any gluing $\left(w_{1}, w_{2}\right)$ that we are given, via a sequence of $\mathrm{R}$ - and $\mathrm{W}$-moves, into a standard gluing:

$$
\left(w_{1}, w_{2}\right) \rightarrow\left(w_{1}^{\prime}, w_{2}^{\prime}\right) \rightarrow\left(w_{1}^{\prime \prime}, w_{2}^{\prime \prime}\right) \rightarrow \cdots \rightarrow(\overrightarrow{1} \overrightarrow{2} \cdots \vec{n}, 2 \downarrow 3 \downarrow \cdots n \downarrow) .
$$

The explanation of why this is true will be illustrated by the example of the gluing $(\overleftarrow{1} \overleftarrow{2} 3 \overleftarrow{4}, 4 \downarrow 2 \downarrow 3 \uparrow)$

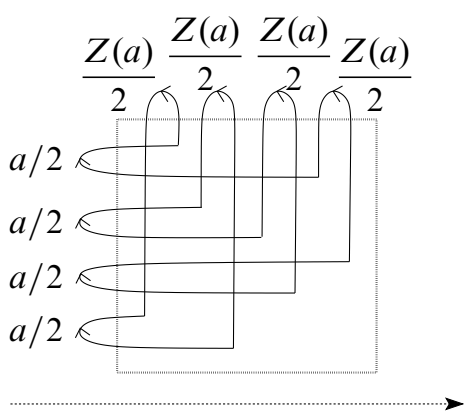

There are two steps in the procedure.

The first step Begin by doing R-moves to put the factors along the top and down the side into the order in which they appear in the words $w_{1}$ and $w_{2}$. Let's go through this in the case of the example shown in line (15). We'll start by swapping row 3 and row 4 , to get

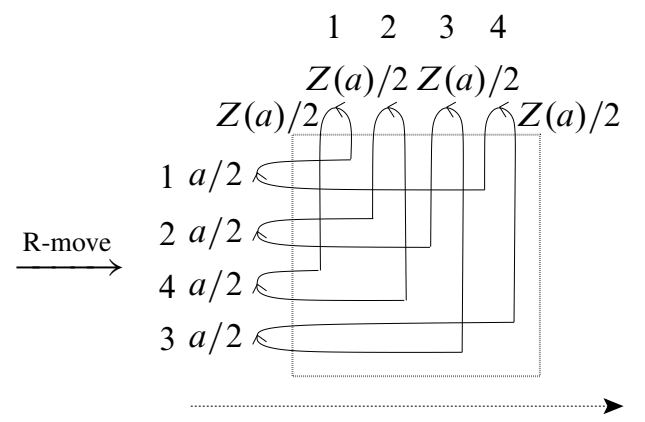

Then we'll swap row 2 and row 3 (ie this swaps factor 2 and factor 4), giving

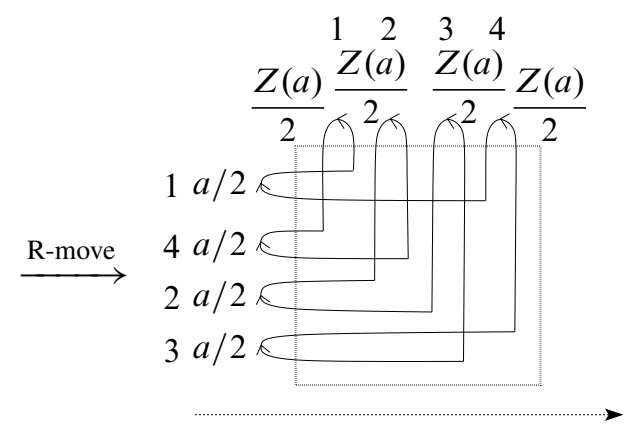


This finishes the first step of the procedure. The thing to notice is that after this step the factors along the top are visited in order from left to right, and the factors down the side are visited in order from top to bottom.

The second step The second step of the procedure is to employ $\mathrm{W}$-moves to arrange it so that, as the drawing is traversed (in the sense indicated in Figure 14), the factors along the top are traversed from left-to-right, and the factors up the side (except the top-most) are traversed from top-to-bottom.

In our example, the diagram can be put in standard position by four such twists. In general: it is clear that these two steps will transform any given $\left(w_{1}, w_{2}\right)$ into the standard gluing, and so, by repeated application of Equation (14), for any $\left(w_{1}, w_{2}\right) \in$ $\vec{\Phi}_{n}$, we have

$$
\phi_{\left(w_{1}, w_{2}\right)}=\frac{1}{n ! n !}(-1)^{x\left(w_{1}, w_{2}\right)} D_{\left(w_{1}, w_{2}\right)}=\frac{1}{n ! n !}(-1)^{x\left(w_{1}^{s}, w_{2}^{s}\right)} D_{\left(w_{1}^{s}, w_{2}^{s}\right)},
$$

where $\left(w_{1}^{s}, w_{2}^{s}\right)=(\overrightarrow{1} \overrightarrow{2} \ldots \vec{n}, 2 \downarrow 3 \downarrow \ldots n \downarrow)$, the standard gluing.

It remains to compute the contribution corresponding to the standard gluing. We'll illustrate the general answer by the specific case $n=4$. The term we need to compute is

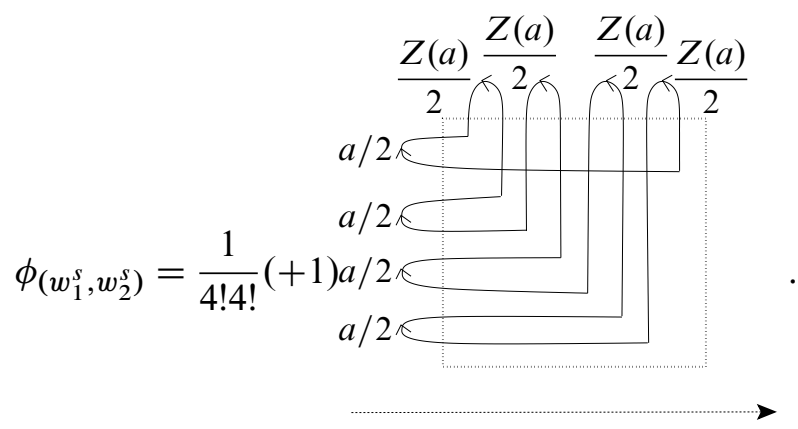

This series is equal to

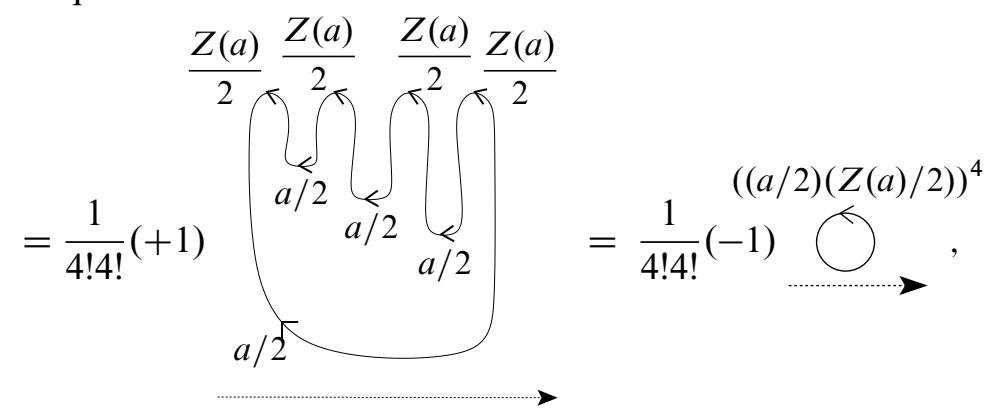

as required. The case of general $n$ follows in the same way. That completes the proof of Lemma 6.2.5. 


\section{References}

[1] A Alekseev, E Meinrenken, The noncommutative Weil algebra, Invent. Math. 139 (2000) 135-172 MR1728878

[2] A Alekseev, E Meinrenken, Lie theory and the Chern-Weil homomorphism, Ann. Sci. École Norm. Sup. (4) 38 (2005) 303-338 MR2144989

[3] D Bar-Natan, On the Vassiliev knot invariants, Topology 34 (1995) 423-472 MR1318886

[4] D Bar-Natan, S Garoufalidis, L Rozansky, DP Thurston, Wheels, wheeling, and the Kontsevich integral of the unknot, Israel J. Math. 119 (2000) 217-237 MR1802655

[5] D Bar-Natan, S Garoufalidis, L Rozansky, D P Thurston, The Århus integral of rational homology 3-spheres. I. A highly non trivial flat connection on $S^{3}$, Selecta Math. (N.S.) 8 (2002) 315-339 MR1931167

[6] D Bar-Natan, T T Q Le, D P Thurston, Two applications of elementary knot theory to Lie algebras and Vassiliev invariants, Geom. Topol. 7 (2003) 1-31 MR1988280

[7] J S Birman, X-S Lin, Knot polynomials and Vassiliev's invariants, Invent. Math. 111 (1993) 225-270 MR1198809

[8] S Garoufalidis, A Kricker, A rational noncommutative invariant of boundary links, Geom. Topol. 8 (2004) 115-204 MR2033481

[9] M Kontsevich, Vassiliev's knot invariants, from: “I M Gel'fand Seminar”, (S Gel'fand, S Gindikin, editors), Adv. Soviet Math. 16, Amer. Math. Soc. (1993) 137-150 MR1237836

[10] A Kricker, Differential operators and wheels power series arXiv:0907.4563

[11] A Kricker, Noncommutative Chern-Weil theory and the combinatorics of wheeling, Duke Math. J. 157 (2011) 223-281

[12] M Nieper-Wißkirchen, Chern numbers and Rozansky-Witten invariants of compact hyper-Kähler manifolds, World Scientific, River Edge, NJ (2004) MR2110899

[13] J Roberts, S Willerton, On the Rozansky-Witten weight systems, Algebr. Geom. Topol. 10 (2010) 1455-1519 MR2661534

Division of Mathematical Sciences, School of Physical and Mathematical Sciences Nanyang Technological University

Singapore 637616, Singapore

ajkricker@ntu.edu.sg

http://www.ntu.edu.sg/home/ajkricker/

Received: 15 December 2009 Revised: 21 December 2010 